\title{
The role of vaccines in combatting antimicrobial resistance
}

Francesca Micoli ${ }^{1}$, Fabio Bagnoli ${ }^{2}$, Rino Rappuoli ${ }^{2 凶}{ }^{2}$ and Davide Serruto ${ }^{2}$

Abstract | The use of antibiotics has enabled the successful treatment of bacterial infections, saving the lives and improving the health of many patients worldwide. However, the emergence and spread of antimicrobial resistance (AMR) has been highlighted as a global threat by different health organizations, and pathogens resistant to antimicrobials cause substantial morbidity and death. As resistance to multiple drugs increases, novel and effective therapies as well as prevention strategies are needed. In this Review, we discuss evidence that vaccines can have a major role in fighting AMR. Vaccines are used prophylactically, decreasing the number of infectious disease cases, and thus antibiotic use and the emergence and spread of AMR. We also describe the current state of development of vaccines against resistant bacterial pathogens that cause a substantial disease burden both in high-income countries and in low- and medium-income countries, discuss possible obstacles that hinder progress in vaccine development and speculate on the impact of next-generation vaccines against bacterial infectious diseases on AMR.

The use of antimicrobials has enabled the treatment of potentially life-threatening infections, saving the lives and improving the health of many patients worldwide. However, the increasing number and global distribution of drug-resistant pathogens is one of the major health challenges, compromising the ability to prevent and cure a wide range of infectious diseases that were once treatable.

The emergence of antimicrobial resistance (AMR) results in drug inefficiency and persistent infections, with a subsequent increase in the risk of severe disease and transmission. Antibiotic resistance is driven by several mechanisms ${ }^{1}$ (Supplementary Box 1) and occurs naturally, but the emergence and spread of new resistance mechanisms may have been greatly accelerated by the overuse and misuse of antimicrobials in the community setting and the hospital setting as well as in the agricultural setting ${ }^{2}$. AMR has become an endemic and widespread problem that affects both high-income countries (HICs) and lowand medium-income countries (LMICs) ${ }^{3,4}$. In the past few years, progress has been made in highlighting AMR as a global health threat, with the "Political Declaration of the High-Level Meeting of the General Assembly on Antimicrobial Resistance" representing a milestone in the commitment to fight $\mathrm{AMR}^{5}$.

Given how fast resistance has evolved to each new class of antibiotic introduced and the challenges in producing new effective drugs, focusing on research into the underlying resistance mechanisms and the development of new antibiotics alone is insufficient ${ }^{6}$. An integrated strategy that includes vaccines together with novel antibiotics, diagnostic tools, monoclonal antibodies, microbiota interventions and the use of bacteriophages is required to combat AMR effectively (BOX 1).

Vaccines have an unprecedented impact on human health ${ }^{7}$ and can be used for decades with a much lower probability of resistance emergence compared with antibiotics $^{8}$.

New technologies and approaches such as reverse vaccinology, novel adjuvants, structural vaccinology, bioconjugates and rationally designed bacterial outer membrane vesicles (OMVs), together with progress in polysaccharide conjugation and antigen design, are promising for the future of vaccine research and development ${ }^{9}$ (BOX 2). Numerous vaccines have been licensed during the past 40 years, and new vaccines against many antimicrobial-resistant bacteria are also being developed.

In this Review, we discuss available data that document the impact of existing vaccines on $\mathrm{AMR}^{10}$ and possible implications of future vaccines that target antimicrobial-resistant pathogens. To this end, we explore the status of vaccine development at the preclinical and clinical stages for a selected list of bacterial pathogens emphasized as critical by the WHO and the CDC (TABLE 1; Supplementary Table 1) that affect both HICs and LMICs. A review of the vaccine development state for all antimicrobial-resistant pathogens is beyond the scope of this Review, and we focus on a select number of resistant bacterial pathogens as examples with the aim to discuss possible obstacles in vaccine development that have led to vaccine failure, and to explore how new technologies can overcome such limitations. 
The AMR crisis and vaccines

AMR emergence and focus on infection prevention. Antimicrobial-resistant infections are already very common, resulting in longer hospital stays and higher medical costs, as well as increased mortality. Global annual deaths from drug-resistant infections have been estimated to be $\sim 700,000$, and the increase of infections with antimicrobial-resistant pathogens may pose a health threat, with the number of deaths exceeding those due to cancer by $2050\left(\right.$ REF. $\left.^{11}\right)$. The current situation is alarming. For example, a study conducted to estimate the incidence of infections due to antimicrobial-resistant pathogens analysed data from 2015 from the European Antimicrobial Resistance Surveillance Network (EARS-Net) and showed a substantial increase of infections with antibiotic-resistant bacteria since 2007 (REF. ${ }^{12}$ ).

The $\mathrm{CDC}^{13}$ and the $\mathrm{WHO}^{14}$ have listed pathogens with concerning drug-resistance patterns. In industrialized

\section{Box 1 Alternative strategies to fight antimicrobial resistance}

The global challenge of antimicrobial resistance needs an integrated strategy to develop new interventions to fight multidrug-resistant bacterial pathogens effectively. Although the focus of this Review is on the role of vaccines, other promising approaches have been considered and published in preclinical and clinical studies (see the figure).

Monoclonal antibodies (mAbs). mAbs have been used as therapeutics for many decades, and can be considered a key strategy to fight emerging infectious diseases and antimicrobial-resistant pathogens ${ }^{136,137}$. mAbs bind to virulence factors that are expressed by bacterial pathogens (for example, polysaccharides, toxins, adhesins or effector proteins) and can act through three main mechanisms: inhibiting the activity of the target; promoting complement-mediated cell lysis; and enabling opsonophagocytosis of bacteria by phagocytic effector cells. There are multiple mAbs to antimicrobial-resistant bacterial pathogens in different stages of development. The most important and most advanced examples are represented by a bispecific $\mathrm{mAb}$ that targets a virulence factor and the exopolysaccharide of Pseudomonas aeruginos $a^{138}$ and a combination of two mAbs targeting Clostridioides difficile toxins $\mathrm{A}$ and $\mathrm{B}^{139}$. One key challenge for using $\mathrm{mAbs}$ to treat bacterial infections is that a $\mathrm{mAb}$ recognizes a single target, whereas diseases caused by bacterial pathogens are usually multifactorial. However, new technologies have enabled the generation of bispecific $\mathrm{mAbs}$ as described for P. aeruginosa, as well as the modification of the Fc portion for the generation of hexamers (through Hexabody technology) that can increase complement activation, as described for Neisseria gonorrhoea $e^{140}$. In the context of antimicrobial-resistant targets, the use of mAbs to protect against hospital-acquired infections, such as P. aeruginosa or Klebsiella pneumoniae infection, in patients at high risk may be a more pragmatic approach than vaccination. A mAb-based approach is attractive as many patients with bacterial infections can be immunocompromised or elderly, and may not mount an effective immune response to vaccines.

Bacteriophages. A common approach for bacterial therapy involves lytic bacteriophages (phages) that enter a productive cycle in which progeny phages are released through bacterial lysis. Specificity, low toxicity towards mammalian cells and the possibility to administer a large number of phages in a very small dose are the key advantages of this approach. Phage therapy has been developed for antimicrobial-resistant bacterial targets, such as Staphylococcus aureus ${ }^{141}$ and P. aeruginos $a^{142}$. In the case of $P$. aeruginosa and $C$. difficile infections, researchers have also explored the possibility of administering phages at the site of infections, such as directly into the lung by inhalation or orally into the gastrointestinal tract ${ }^{143,144}$. Phages can be stabilized through adsorption or encapsulation and, moreover, could be used as CRISPR-Cas delivery systems in bacteria ${ }^{145}$.

Microbiota. The human microbiota has a major impact on the health of the host and its immune response ${ }^{146}$. Antibiotics not only target pathogens but can also eliminate the commensal bacterial community, which may provide an opportunity for opportunistic bacteria to colonize the human host and cause infections. In the context of antimicrobial-resistant bacterial pathogens, examples to treat (recurrent) C. difficile-associated diarrhoea and re-establish the gut microbiota have been described: oral administration of a mixture of spores from several bacteria isolated from faecal samples from a healthy donor has shown promising results in preventing reinfection ${ }^{147}$; and administration of a

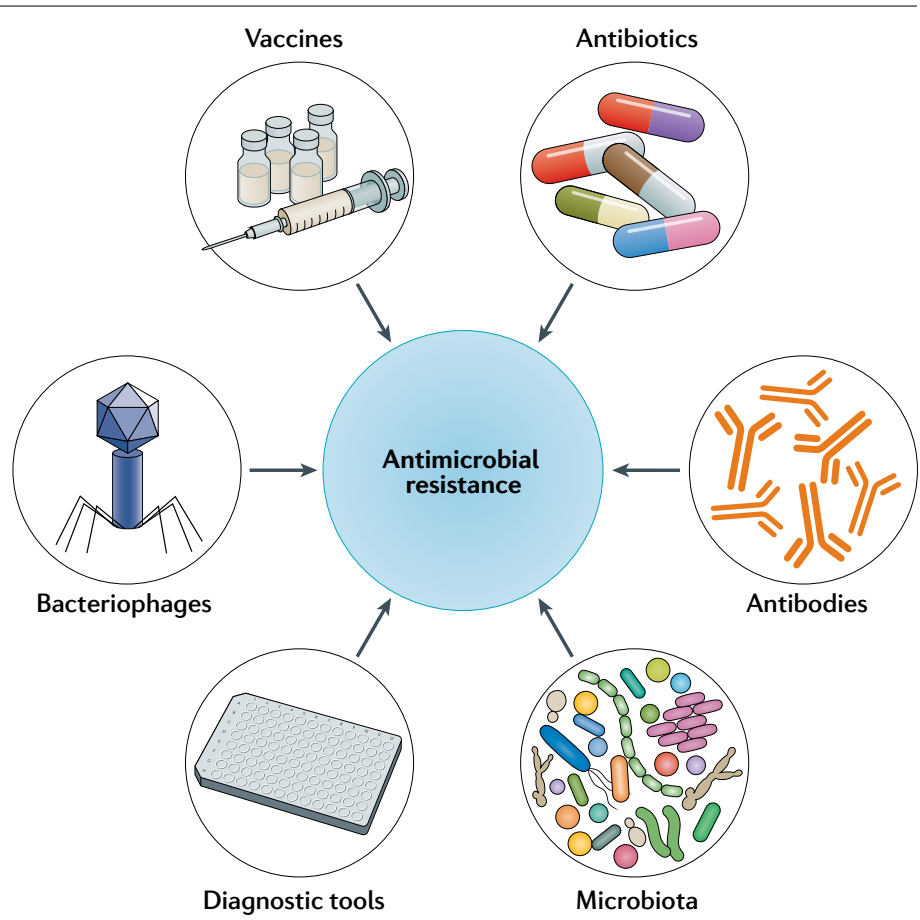

non-toxigenic $C$. difficile that can outcompete the infecting toxic C. difficile resulted in a significant reduction in C. difficile infection recurrence and the possibility to restore the microbiota ${ }^{148}$. In addition to the gut microbiota, other microbiota-based intervention strategies might in the future be applied to prevent respiratory infections or sexually transmitted diseases ${ }^{149}$.

Diagnostic tools. Diagnostic tools are used to identify and characterize the causative agents of microbial infections, and to generate antimicrobial susceptibility profiles that can inform the treatment strategy. Antimicrobial susceptibility testing (AST) can be performed through phenotypic and genotypic methods ${ }^{150}$. AST is usually time-consuming, and it takes up to 48 hours for the identification of the causative agent and for the release of a complete and validated resistance profile that then allows the prescription of an appropriate therapy ${ }^{151}$. State-of-the-art techniques (for example, flow cytometry or mass spectrometry) are being explored for the development of more rapid AST, and some progress has been described ${ }^{152}$. However, reliable diagnostic tools for some pathogens still do not exist or are not accessible in some global geographical regions and, therefore, in most cases infections are treated without isolating or serotyping the infecting microorganism. For example, lack of appropriate diagnostic tools, particularly in Africa, hampers effective management of invasive non-typhoidal salmonellosis. Currently, these infections can be detected only by microbial culture, and facilities able to perform such tests are rare in developing countries ${ }^{73}$. The development of sustainable and rapid diagnostic tools is a priority in the context of antimicrobial resistance that will help to prevent inappropriate prescriptions and enable the use of targeted and effective antibiotics worldwide. 
Basic technologies for vaccine development relied on growing bacteria and viruses and on developing vaccines by killing them, attenuating them or purifying immunogenic components. Genetic engineering has given scientists the ability to rationally design and produce both individual microbial components and whole microorganisms. Glycoconjugation enables the covalent linking of a bacterial polysaccharide to a carrier protein and has provided successful vaccines licensed worldwide against Haemophilus influenzae, meningococcus serotypes C, A and ACWY, pneumococcus serotypes 7,10 and 13 and Salmonella enterica subsp. enterica serovar Typhi $\mathbf{i}^{153}$. Substantial scientific progress in genomics, bioinformatics, genetics, microbiology, immunology and structural biology has provided a new set of tools to approach vaccine development for many unmet medical needs, including antimicrobial-resistant bacterial pathogens ${ }^{9,154}$.

Reverse vaccinology. Reverse vaccinology enables the selection of potential vaccine candidates on the basis of the genomic information of a bacterial strain. The complete genome of a bacterium represents the catalogue of genes that encode potential antigens that can be selected, screened and tested as vaccine candidates in both in vitro and in vivo preclinical models. Therefore, potentially surface-exposed immunogenic proteins can be identified in a reverse manner ${ }^{155}$. This approach was used for the development of a meningococcus $B$ vaccine ${ }^{156}$ that was shown to be highly effective in preventing meningococcal disease. A similar genome-based antigen selection approach has been described for the development of candidate vaccines against Escherichia coli ${ }^{157}$ and Pseudomonas aeruginosa ${ }^{63}$.

Structural vaccinology. Structural information combined with immunological and functional characterization of microbial antigens can be used to structurally design new protective and effective vaccine antigens. The possibility to isolate protective human monoclonal antibodies (from patients who are infected or vaccinated), combined with the ability to determine the structure of antigens and antigenantibody complexes, can be used to engineer improved antigens ${ }^{158}$; this approach takes advantage of the greatly enhanced ability to clone human $B$ cells and then to produce the corresponding recombinant monoclonal antibody or antigen-binding fragments. A specific conformation of the respiratory syncytial virus $F$ protein was stabilized to elicit a strong functional protective response both in animals and in humans ${ }^{159}$. Although most of the applications reported so far target viral antigens, the same technologies can be applied for bacterial proteins to either rationally develop cross-protective antigens ${ }^{160}$ or display antigens on nanoparticles to increase their immunogenicity. To fully exploit B cell technology and structural biology in vaccine design for antimicrobial-resistant bacterial pathogens, it will be key to have access to sera from patients who are infected to fully integrate $B$ cell analysis in early clinical studies for vaccine antigens under development and to have functional assays to screen protective monoclonal antibodies.

Generalized modules for membrane antigens (GMMA). GMMA are outer membrane vesicles generated from Gram-negative bacterial strains that have been genetically modified to enhance release of outer membrane vesicles. This approach is generally aimed at disrupting the anchorage of the outer membrane to the peptidoglycan. Naive vesicles contain natural bacterial surface-exposed proteins in the correct conformation, and therefore have the potential to be more protective when used as vaccine components. In addition, genetic manipulation can be used to reduce lipopolysaccharide-mediated reactogenicity (for example, by targeting genes responsible for lipid A acylation), to display and/or overexpress homologous or heterologous antigens (proteins or glycans) and to delete unwanted interfering antigens ${ }^{161}$. GMMA is a promising powerful platform for generating vaccines against Gram-negative antimicrobial-resistant bacterial pathogens; for example, a Shigella sonnei GMMA-based vaccine has been shown to be well tolerated and immunogenic in preclinical and clinical studies $^{92,162-164}$.

Bioconjugation. Recently, a simple way to generate glycoconjugates in vivo was proposed for vaccine development. The oligosaccharyltransferase PglB exhibits relaxed substrate specificity towards glycans and covalently links polysaccharides to target carrier proteins that contain specific $\mathrm{N}$-glycosylation sites ${ }^{165}$. A single $E$. coli strain can be genetically engineered to express all the elements needed to produce glycoconjugate molecules in the bacterial periplasm: enzymes that synthesize the specific polysaccharide, the carrier protein and the oligosaccharyltransferase PglB. Through this new process a glycoconjugate vaccine can be produced in a single fermentation step, and it is possible to use a protective antigen as a carrier without interfering with protective epitopes because glycosylation sites can be rationally positioned in the amino acid sequence. Bioconjugates may be able to prevent infections caused by antimicrobial-resistant pathogens that express polysaccharide antigens, such as Shigella flexneri, Staphylococcus aureus, P. aeruginosa and Klebsiella pneumoniae ${ }^{89,166}$.

Adjuvants. Adjuvants have traditionally been used to improve the immune response elicited by a vaccine. Several novel adjuvants, including AS01, AS03, AS04 and other Toll-like receptor agonists, have been described and licensed in new vaccines ${ }^{167}$. The most notable example is AS01, which was recently licensed for a vaccine against malaria and for a novel vaccine against herpes zoster ${ }^{168,169}$. This adjuvant comprises a mix of liposomes that contain saponin QS21 and monophosphoryl lipid A (MPL) which exert the synergistic effect that is usually obtained by combining different immunostimulants. The AS01 adjuvant has also been used in an investigational subunit vaccine designed to prevent reactivation of tuberculosis which showed $54 \%$ efficacy in clinical studies ${ }^{110,111}$. countries, the AMR problem is often claimed to be associated with hospital-acquired infections ${ }^{15}$; that is, infections occurring after surgery (for example, surgical site infections and bloodstream infections), catheter-associated infections (for example, bloodstream infections), gastrointestinal infections (for example, colitis caused by Clostridioides difficile), and intensive care unit-associated infections (for example, ventilator-associated pneumonia caused by Staphylococcus aureus, Pseudomonas aeruginosa and Klebsiella pneumoniae). However, community-acquired infections are much more frequent than hospital-acquired infections and affect a much larger population ${ }^{16,17}$. For example, skin and soft tissue infections, respiratory infections (including pneumonia), urinary tract infections (UTIs) and gastrointestinal infections are very common in the community setting (for example, approximately 11 million to 14 million visits to physician offices, hospital outpatient departments and emergency departments per year in the USA are due to skin and soft tissue infections ${ }^{16}$ ). Many of these infections are treated with antibiotics, which suggests that community-acquired infections are an important driver of resistance emergence and spread of antimicrobial-resistant pathogens. For instance, the multidrug-resistant $S$. aureus strain USA300 was initially prevalent in the community, but it then spread into the hospital setting, which provides insights into emergence, distribution and transmission dynamics of the resistant pathogen ${ }^{17}$.

The AMR crisis is often perceived as a priority for HICs only ${ }^{18,19}$. However, pathogens that are highly 
Table 1 | Overview of the antimicrobial-resistant pathogens described in this Review

\begin{tabular}{|c|c|c|c|c|c|c|}
\hline Pathogen & $\begin{array}{l}\text { Main diseases } \\
\text { caused }\end{array}$ & $\begin{array}{l}\text { Annual global } \\
\text { mortality (annual } \\
\text { deaths per } 1,000)^{a}\end{array}$ & $\begin{array}{l}\text { Antibiotics (a group or } \\
\text { a specific compound) } \\
\text { for which resistance } \\
\text { has been reported }\end{array}$ & CDC priority & WHO priority & Refs \\
\hline difficile & $\begin{array}{l}\text { Diarrhoea and } \\
\text { colitis }\end{array}$ & 12.8 (USA only) $^{\mathrm{b}}$ & $\begin{array}{l}\text { Aminoglycosides, } \\
\beta \text {-lactams, tetracyclines, } \\
\text { macrolides, glycopeptides } \\
\text { and quinolones }\end{array}$ & Urgent & Not listed & $13,120,121$ \\
\hline \multirow{2}{*}{$\begin{array}{l}\text { Staphylococcus } \\
\text { aureus }\end{array}$} & \multirow{2}{*}{$\begin{array}{l}\text { SSI, BSI, SSTI } \\
\text { and pneumonia }\end{array}$} & \multirow{2}{*}{$\begin{array}{l}10.6 \text { (methicillin- }^{-} \\
\text {resistant strains, } \\
\text { USA only) }\end{array}$} & \multirow{2}{*}{$\begin{array}{l}\beta \text {-Lactams, aminoglycosides, } \\
\text { tetracyclines, macrolides, } \\
\text { glycopeptides, quinolones, } \\
\text { lipopeptide and } \\
\text { oxazolidinone }\end{array}$} & Serious (MRSA) & \multirow[t]{2}{*}{ High } & \multirow[t]{2}{*}{$13,14,28,49,123$} \\
\hline & & & & Concerning (VRSA) & & \\
\hline $\begin{array}{l}\text { Pseudomonas } \\
\text { aeruginosa }\end{array}$ & $\begin{array}{l}\text { Pneumonia, UTI } \\
\text { and SSI }\end{array}$ & $\begin{array}{l}2.7 \text { (multidrug-resistant } \\
\text { strains, USA only) }\end{array}$ & $\begin{array}{l}\beta \text {-Lactams, } \\
\text { aminoglycosides, } \\
\text { quinolones and polymyxins }\end{array}$ & Serious & Critical & $13,14,125$ \\
\hline $\begin{array}{l}\text { Klebsiella } \\
\text { pneumoniae }\end{array}$ & $\begin{array}{l}\text { Pneumonia, } \\
\text { meningitis, UTI } \\
\text { and BSI }\end{array}$ & $\begin{array}{l}1.1 \text { (carbapenem- } \\
\text { resistant } \\
\text { Enterobacteriaceae, } \\
\text { USA only) }\end{array}$ & $\begin{array}{l}\beta \text {-Lactams (including } \\
\text { carbapenems), } \\
\text { aminoglycosides and } \\
\text { fluoroquinolones }\end{array}$ & $\begin{array}{l}\text { Urgent } \\
\text { (Enterobacteriaceae) }\end{array}$ & $\begin{array}{l}\text { Critical } \\
\text { (Enterobacteriaceae) }\end{array}$ & $13,14,126$ \\
\hline $\begin{array}{l}\text { Non-typhoidal } \\
\text { Salmonella }\end{array}$ & $\begin{array}{l}\text { Gastrointestinal } \\
\text { disease in } \\
\text { HICs; BSIs in } \\
\text { sub-Saharan } \\
\text { Africa }\end{array}$ & 59 & $\begin{array}{l}\beta \text {-Lactams, sulfonamides, } \\
\text { chloramphenicol and } \\
\text { fluoroquinolones }\end{array}$ & Serious & $\begin{array}{l}\text { High } \\
\text { (Salmonella spp.) }\end{array}$ & $13,14,129,130$ \\
\hline Shigella species & $\begin{array}{l}\text { Moderate } \\
\text { to severe } \\
\text { diarrhoea }\end{array}$ & 238 & $\begin{array}{l}\text { Sulfonamides, } \\
\text { fluoroquinolones, } \\
\text { macrolides, } \beta \text {-lactams } \\
\text { and cephalosporins }\end{array}$ & Serious & Medium & $13,14,131$ \\
\hline \multirow{2}{*}{$\begin{array}{l}\text { Group A } \\
\text { Streptococcus }\end{array}$} & \multirow{2}{*}{$\begin{array}{l}\text { Pharyngitis and } \\
\text { skin infections; } \\
\text { PSGN, ARF } \\
\text { and RHD }\end{array}$} & 285 (RHD) & \multirow{2}{*}{$\begin{array}{l}\text { Tetracycline and } \\
\text { macrolides }\end{array}$} & \multirow[t]{2}{*}{ Concerning } & \multirow[t]{2}{*}{ Not listed } & \multirow[t]{2}{*}{$13,132,133$} \\
\hline & & $\begin{array}{l}5.4 \text { (erythromycin- } \\
\text { resistant strains, } \\
\text { USA only) }\end{array}$ & & & & \\
\hline \multirow{2}{*}{$\begin{array}{l}\text { Mycobacterium } \\
\text { tuberculosis }\end{array}$} & \multirow{2}{*}{$\begin{array}{l}\text { Predominantly } \\
\text { pulmonary } \\
\text { disease }\end{array}$} & 1,184 & \multirow{2}{*}{$\begin{array}{l}\beta \text {-Lactams, } \\
\text { fluoroquinolones, } \\
\text { aminoglycosides, } \\
\text { macrolides, lincosamides, } \\
p \text {-aminosalicylic acid } \\
\text { and pyrazinamide }\end{array}$} & \multirow[t]{2}{*}{ Serious } & \multirow{2}{*}{$\begin{array}{l}\text { Not listed because it } \\
\text { is already a globally } \\
\text { established priority } \\
\text { pathogen }\end{array}$} & \multirow[t]{2}{*}{$13,14,134,135$} \\
\hline & & $\begin{array}{l}62 \text { (drug-resistant } \\
\text { strains, USA only) }\end{array}$ & & & & \\
\hline
\end{tabular}

The bacterial pathogens emphasized as critical by the WHO and CDC were selected as examples with the aim to discuss possible obstacles in vaccine development and to explore how new technologies can overcome such limitations. ARF, acute rheumatic fever; BSI, bloodstream infection; HICs, high-income countries; MRSA, methicillin-resistant S. aureus; PSGN, post-streptococcal glomerulonephritis; RHD, rheumatic heart disease; SSI, surgical site infection; SSTI, skin and soft tissue infection; UTI, urinary tract infection; VRSA, vancomycin-resistant S. aureus. ${ }^{\mathrm{G} G l o b a l}$ mortality (annual deaths per 1,000) based on Global Burden of Disease data 2017 from the Institute for Health Metrics and Evaluation and data from REF. ${ }^{44}$. ${ }^{\circ}$ Estimated deaths in the USA (annual deaths per 1,000) in 2017 (REF. ${ }^{13}$. 
prevalent in LMICs have a concerning resistance profile. For example, $45 \%$ of deaths in Africa and South-East Asia were due to antimicrobial-resistant bacteria, and most of the pathogens responsible have the same prevalence in HICs (resistant K. pneumoniae, Acinetobacter baumannii, $P$. aeruginosa and $S$. aureus are among the most common bacteria associated with increased mortality both in HICs and in LMICs) ${ }^{20}$. An additional problem in the developing world is that the use of antibiotics is often uncontrolled and inappropriate due to their availability without prescription ${ }^{21}$.

Unfortunately, scientific challenges, clinical and regulatory hurdles and low return on investment led many companies to disinvest from research and development of new antibiotics ${ }^{22}$. Indeed, recent efforts in identifying new compounds based on high-throughput genome screening led to the identification of only a few potential candidates, and validation of their antibiotic activity and druggability was in most cases impossible ${ }^{9}$.

This situation indicates two important needs: new and sustainable investments in antibiotic research and development (recently demonstrated by the creation of the AMR Action Fund) and alternative medical interventions against antimicrobial-resistant pathogens. Disease prevention is an important measure to combat AMR, and vaccines could not only prevent or reduce life-threatening diseases and thus decrease health care costs and sequelae remaining after infection resolution but could also reduce the use of antibiotics (both first-line and second-line drugs), with the potential of decreasing the emergence of AMR (FIG. 1a). If sufficient vaccine coverage is achieved in a population, indirect protection (herd immunity) further prevents the spread of resistant strains (FIG. 1 b).

The mechanisms of action of antibiotics and vaccines linked to the generation of resistance are intrinsically different (FIG. 2) and may account for the lower probability of resistance emergence for vaccines.

Vaccines are used prophylactically and are thus effective before bacteria start to multiply following the initial infection (low pathogen burden) and before different tissues and organs are affected, which substantially reduces the likelihood that resistance-conferring mutations will emerge and spread $^{22}$. In addition, whereas antibiotics have a single target (such as the bacterial cell wall or the translation machinery), vaccines usually contain multiple immunogenic epitopes. Thus, more mutations are necessary to confer resistance against a vaccine (FIG. 2). Although resistance is probably less likely to emerge for vaccines, there are notable examples of vaccine resistance. Emergence of vaccine resistance is exemplified by hepatitis B virus ${ }^{23}$. Recombinant DNA vaccines that target the surface antigen present on the outer protein coat of the virus (hepatitis B surface antigen (HBsAg)) were commercialized in $1986\left(\mathrm{REF}^{24}{ }^{24}\right.$. Neutralizing antibodies bind mainly to a hydrophilic amino acid region spanning amino acids 124-149 of HBsAg, referred to as the common determinant. A substitution of arginine for glycine at position 145 (G145R) within the determinant region of $\mathrm{HBs} A g$ resulted in the inability of neutralizing antibodies to recognize this antigen. Since then, breakthrough infections caused by variants of the virus with mutations in the gene encoding HBsAg have occasionally been reported ${ }^{23}$. However, hepatitis B virus infection in vaccinated people remains rare, and the vaccine has almost eradicated the virus from many countries ${ }^{22}$. Another potential example of the emergence of vaccine resistance has been reported for Bordetella pertussis ${ }^{25}$. Vaccination against this pathogen has greatly reduced the incidence of whooping cough. However, lately the disease has re-emerged ${ }^{26}$, and the reason for this is debated $^{8}$. One possibility is the emergence of vaccine escape mutants. Indeed, genetic variability in the two key antigens pertactin and pertussis toxin has been observed in bacterial strains that circulate after the vaccine has been introduced ${ }^{27}$. However, it has also been proposed that the increase in disease incidence is driven mainly by epidemiological and immunological factors ${ }^{8,28}$.

Moreover, if vaccine coverage is limited, serotypes not included in the vaccine can emerge owing to selective pressure (serotype replacement). For example, after the introduction of the heptavalent pneumococcal vaccine (PCV7), an increase in serotype 19A (which is not included in the vaccine) was observed in patients ${ }^{29,30}$.

Fortunately, in the rare instances in which resistance to vaccines has been detected, a reduction in disease burden has still been achieved, mainly due to the preventive nature of vaccination, herd immunity and a durable protective effect ${ }^{8,22,28}$. This is an interesting difference with antibiotics, for which the therapeutic effect in a patient can be completely invalidated by the emergence of resistance.

Direct and indirect effects of vaccines on AMR. Vaccines can reduce the emergence and spread of AMR both directly and indirectly ${ }^{10,28}$. First, a vaccine against a given bacterial pathogen reduces prevalence of the resistant pathogen as well as antibiotic use. Probably the best documented example of this effect is the pneumococcal vaccine. Several studies suggest that decreased pathogen carriage and infections in vaccinees substantially reduced antibiotic prescriptions and diminished the circulation of resistant strains ${ }^{31}$. These findings suggest that herd immunity is a key mechanism in reducing the circulation of antimicrobial-resistant pneumococcal strains ${ }^{32}$. Also, the introduction of Haemophilus influenzae type b conjugate vaccine reduced the need for antibiotics and avoided the continued evolution of resistance, as indicated by data from India, where the introduction of $H$. influenzae type $b$ vaccine was delayed ${ }^{33}$. The veterinary and agricultural settings account for more than $50 \%$ of global antibiotic consumption ${ }^{34,35}$, which has been reported to be an important driver of the emergence of resistance ${ }^{36}$. Recently, it was demonstrated that use of vaccines in food-producing animals substantially decreased antibiotic use and reduced the risk of the emergence of antibiotic resistance ${ }^{37}$. This might also have implications for human health as resistance determinants might be transferred to bacteria that infect humans or resistant pathogens might infect humans directly. However, more studies are needed to confirm this.

Furthermore, vaccinations indirectly affect AMR by preventing viral infections. For example, influenza vaccines can reduce the inappropriate use of antibiotics 


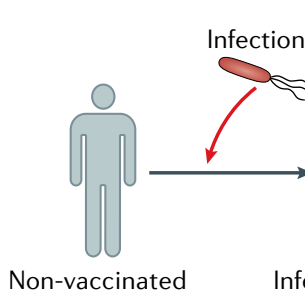

Non-vaccinated patient
Infected patient

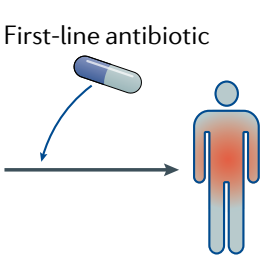

Infection not cleared owing to AMR

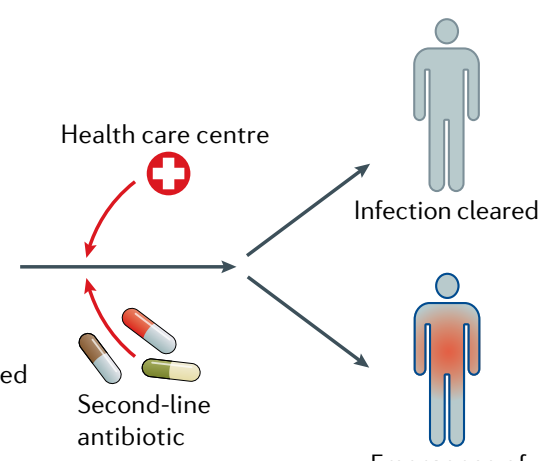

Emergence of antimicrobial-resistant isolates

\section{Population}
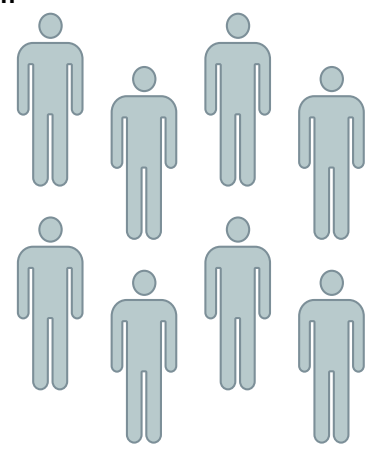

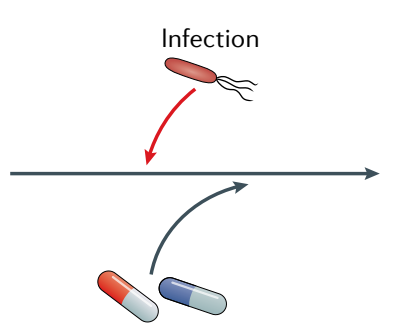

First-line and second-line antibiotics

\section{b}

Single individual

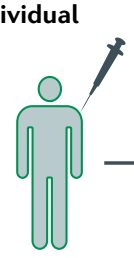

Vaccinated individual
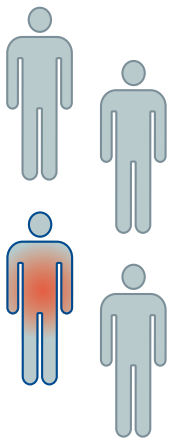

0

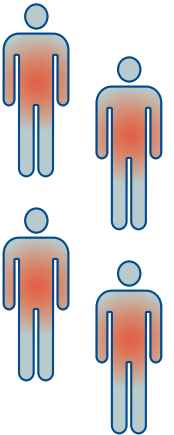

Widespread emergence and spread of antimicrobial-resistant isolates

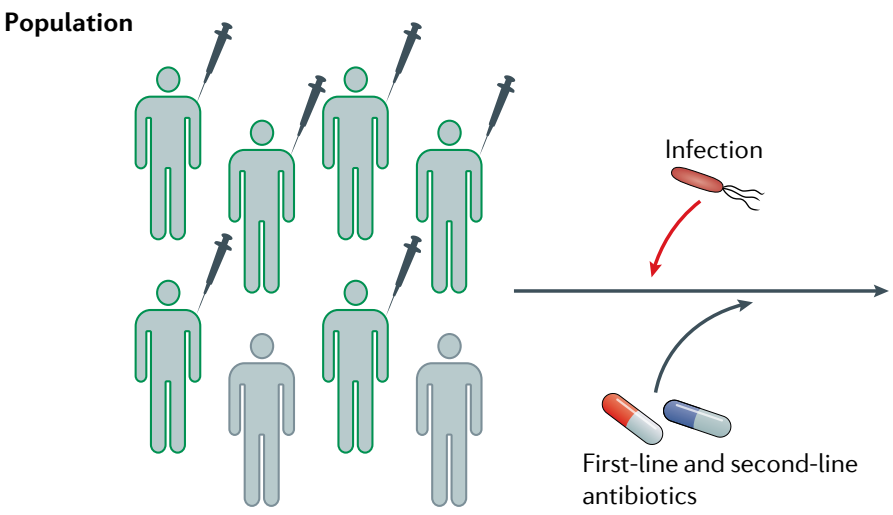

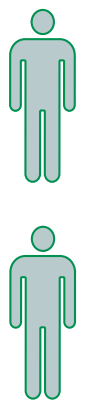
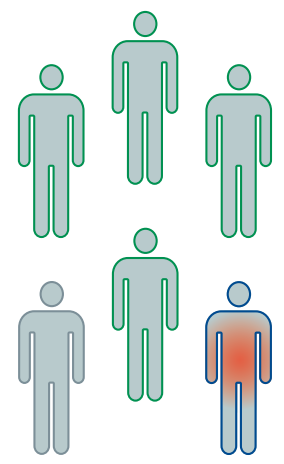

Emergence and spread of AMR are mitigated by vaccination and herd immunity

Fig. 1 | Effects of vaccines on antimicrobial resistance. a | Antimicrobial-resistant bacterial pathogens can cause serious, potentially life-threatening infections in individuals. Treatment with currently available first-line antibiotics is ineffective against resistant infections, and second-line antibiotics may be required to resolve the infection. However, use of the second-line antibiotic may promote the emergence of new antimicrobial-resistant isolates resistant to second-line antibiotics. At the population level, the emergence and spread of antimicrobial resistance (AMR) consequently leads to difficulties in treating patients who are infected. Pathogens resistant to antimicrobials cause substantial morbidity and death. $\mathbf{b}$ | Vaccines against antimicrobial-resistant pathogens could prevent or reduce life-threatening diseases and thus decrease health care costs, and also reduce the use of antibiotics (both first-line and second line drugs) with the potential of decreasing the emergence of AMR. If sufficient vaccine coverage is achieved in a population, indirect protection (herd immunity) further prevents spread of resistant strains. Decreased disease burden would also negate the need for antibiotics. 

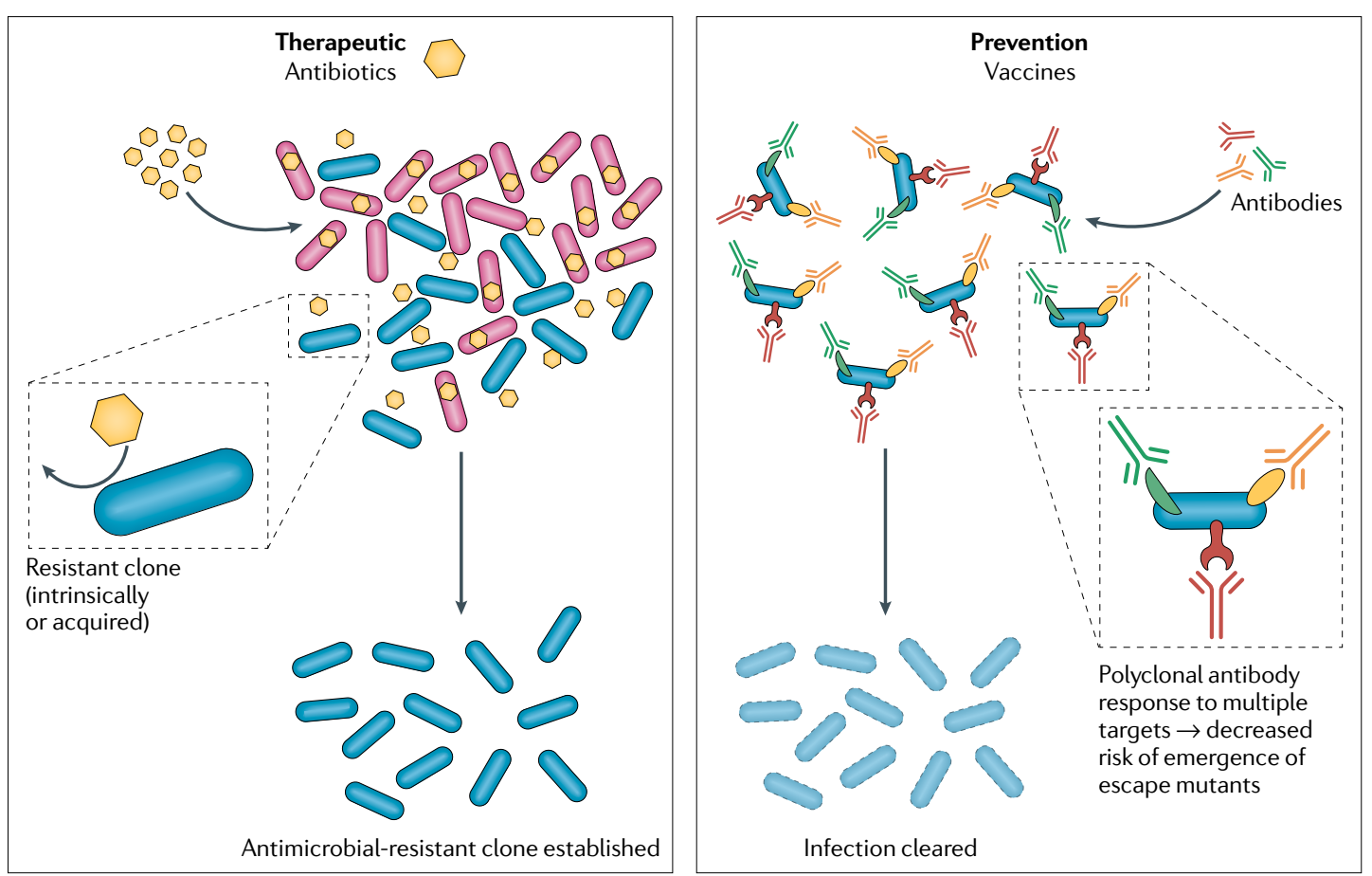

Fig. 2 | Mechanisms of action of antibiotics and vaccines and emergence of resistance. Antibiotics, which are most commonly administered therapeutically, act on established infections against many bacteria, increasing the probability that resistant clones emerge. Antibiotics usually have a single mechanism of action; that is, a single target, such as the bacterial cell wall or the translation machinery. Bacteria either are intrinsically resistant or acquire and/or develop antibiotic resistance (resistance mechanisms include preventing access to antibiotic targets, drug efflux, changes in the drug targets and modification or inactivation of the antibiotic itself). Thus, for example, changes in the drug target by a single mutation render the antibiotic ineffective. In addition, selective pressure exerted by antibiotics favours the emergence of resistant clones. Vaccines, by acting in a preventive manner, decrease the probability that resistant clones are selected. Vaccines often target multiple antigens and/or multiple epitopes of the same antigen (polyclonal antibodies), and thus the emergence of vaccine escape variants would require several mutations impacting different epitopes. However, it is possible that resistant clones emerge through mutations or by serotype replacement.

and prevent secondary bacterial superinfections that may occur in a patient who has been infected with the influenza virus ${ }^{10}$. Indeed, several studies showed reduction in antibiotic prescriptions ranging from about $13 \%$ to $64 \%\left(\right.$ REF. $\left.^{10}\right)$.

In some cases, vaccines have led to the eradication of pathogens, such as the global eradication of smallpox and the animal pathogen rinderpest virus ${ }^{37}$, and the almost complete elimination of poliomyelitis, as well as a decrease of more than $95 \%$ in the incidence of diseases such as diphtheria, tetanus, pertussis, measles, mumps and rubella $^{28}$. Although formal studies to quantify the impact of these vaccines on reducing AMR have not been performed, it is plausible to assume an important contribution through indirect mechanisms by reducing antibiotic use and therefore selection pressure on pathogens.

Unfortunately, vaccines against major antimicrobialresistant pathogens are still missing. However, predictions of the impact of vaccines against antimicrobial-resistant pathogens suggest that vaccines could have a substantial impact in controlling resistance ${ }^{38}$.

\section{Vaccines under development}

In this section, we describe the state of vaccine development at the preclinical and clinical stages for a selected list of pathogens, among the ones emphasized as critical for AMR by the WHO and CDC (TABLE 1), providing a perspective on the state of vaccine development and possible obstacles. Some of the selected pathogens, for example Shigella species and Salmonella species, affect mainly LMICs, where the implementation of existing vaccines is difficult and the absence of commercial incentives is a complication for the development of new vaccines. A description of the pathogens, disease burden, epidemiology, current intervention and treatment options, and resistance emergence can be found in TABLE 1 and Supplementary Box 2.

Clostridioides difficile. At present, no vaccines against C. difficile are available on the market. Vaccines that target the major pathogenic factors toxin A and toxin B (TcdA and TcdB, respectively) are in clinical development. Sanofi started a phase III clinical trial in individuals older than 50 years who are at risk of $C$. difficile infection to assess the efficacy to prevent primary symptomatic episodes. However, the trial was terminated after review of interim data by an independent data monitoring committee, who concluded that the probability that the study would have met its efficacy objective was $\operatorname{low}^{39}$. Pfizer is currently testing a genetically modified full-length TcdA and TcdB toxoid vaccine in a phase III clinical trial ${ }^{40}$. The trial enrolled adults aged 50 years or 
older who are at risk of developing $C$. difficile infection with the aim to assess whether the vaccine prevents the disease. Valneva has completed a phase II trial with a vaccine (VLA84) containing a fusion protein of truncated forms of $\mathrm{TcdA}$ and $\mathrm{TcdB}^{41}$. Both vaccines were highly immunogenic and induced antibodies that neutralized the toxins. GlaxoSmithKline (GSK) recently started a phase I trial assessing safety and immunogenicity of an investigational vaccine based on the F2 antigen $^{42}$. The vaccine is formulated with or without the adjuvant AS01B and is administered to healthy adults aged $18-45$ years and $50-70$ years.

Whether vaccines that target only TcdA and TcdB are optimal candidates is a subject of debate. Indeed, toxin-targeting antibodies might block disease, but they do not reduce the ability of the pathogen to colonize the intestine. For this reason, vaccines that target surface antigens involved in colonization and spore formation are being explored, but are still at the preclinical stage $\mathrm{e}^{43}$ (Supplementary Table 1).

Escherichia coli. Except for whole-cell-based vaccines with suboptimal properties, such as Solco-Urovac (an inactivated polymicrobial vaccine) and Uro-Vaxom (composed of membrane proteins of 18 strains), which are not widely used, no vaccines for extraintestinal pathogenic Escherichia coli (ExPEC) are currently available on the market, and only a few candidates are in development (Supplementary Table 1). A vaccine that is being developed by Sequoia Sciences consists of the bacterial adhesin protein FimH and an adjuvant, and was tested in a phase I clinical trial that enrolled 67 women, with 30 of them having a 2-year documented history of recurrent UTI. Preliminary results suggest that the vaccine may reduce the frequency of $\mathrm{UTI}^{44}$.

Janssen Vaccines, in collaboration with LimmaTech Biologics, is developing a vaccine (ExPEC4V) based on $\mathrm{O}$ antigens that correspond to four prevalent serotypes ${ }^{45}$. The vaccine, produced through the bioconjugation process, was tested in a phase Ib multicentre trial enrolling healthy women with a history of recurrent UTI. In this trial, ExPEC4V was safe and well tolerated, and elicited strong, durable and functional immune responses. Although the study was underpowered to detect a significant reduction in the incidence of UTIs caused by the vaccine-specific serotypes, a decrease in the incidence of UTIs caused by E. coli of any serotype was observed ${ }^{46}$.

Future research efforts should consider the identification of new targets involved in both cystitis and sepsis, given these are the infection outcomes with the greatest unmet medical need. However, development of such a vaccine is complex and will likely require protective efficacy evidence obtained in independent trials with participants affected by cystitis and sepsis. It will also be key to expand research for the identification of factors expressed by pathogenic E. coli and not by commensal E. coli to avoid a potential detrimental effect against the gut flora.

Staphylococcus aureus. Three candidate vaccines against S. aureus have been evaluated for efficacy in clinical trials. StaphVAX, a conjugate vaccine, developed by Nabi Biopharmaceuticals, targeting capsular polysaccharides type 5 (CP5) and CP8 failed to show efficacy in terms of reduction of $S$. aureus bacteraemia in individuals with end-stage renal disease who received haemodialysis ${ }^{47}$. V710, a vaccine targeting the iron-scavenging protein IsdB, developed by Merck, was tested in patients undergoing cardiothoracic surgery in a phase IIb and phase III study to evaluate the efficacy of the vaccine in reducing the proportion of patients with postoperative $S$. aureus bacteraemia and/or deep sternal wound infections. The trial was stopped after an interim analysis showing a low probability of achieving vaccine efficacy as well as for safety concerns ${ }^{48}$.

Pfizer advanced its four-component vaccine candidate SA4ag (containing CP5, CP8 and the two surface protein antigens ClfA and $\mathrm{MntC}$ ) to a phase IIb trial. The trial enrolled patients undergoing elective open posterior multilevel spinal surgery and evaluated the efficacy of the vaccine against postoperative $S$. aureus bloodstream infections and/or deep incisional or organ/space surgical site infections. The trial was discontinued due to an analysis conducted at a preplanned interim observation which suggested low statistical probability for the study to meet the predefined primary efficacy end points.

New promising vaccine candidates such as the virulence factor $\mathrm{SpA}$ and the pore-forming toxins leukocidins as well as novel adjuvants that stimulate cell-mediated immunity and increase vaccine efficacy have been identified and are in the preclinical phase of development ${ }^{49}$ (Supplementary Table 1).

Critical aspects to consider for the clinical development of new vaccine candidates include the selection of suitable target populations for efficacy trials and biomarkers for identifying correlates of protection. Indeed, lack of a known correlate of protection is a major limitation in the ability to identify protective vaccine candidates. The higher risk of severe infections in certain populations (for example, individuals undergoing elective surgery or patients receiving haemodialysis) suggests that a niche vaccination approach limited to those populations would be more cost-effective. However, such a narrow approach would leave a substantial unmet medical need associated with several other hospitalacquired and community-acquired infections and would probably not have a significant effect on decreasing emergence and spread of resistance. Therefore, for this pathogen a mass-vaccination approach would probably provide a higher return on the investment and benefit at the population level. S. aureus is an important pathogen in both developed and developing countries; however, little is known about the incidence and burden of $S$. aureus in LMICs. A better understanding of the epidemiology and disease burden at a global level with a specific focus on the burden of $S$. aureus infection in LMICs will help to increase awareness of the disease and to estimate the cost-effectiveness of a $S$. aureus vaccine.

Neisseria gonorrhoeae. No vaccine against Neisseria gonorrhoeae is currently available, and vaccine development has proven complicated in the past few decades for the following main reasons: $N$. gonorrhoeae surface proteins are subject to antigenic diversity and phase variation; knowledge of the type of immune 
responses that are needed to protect individuals is lacking as is information on reliable correlates of protection; and preclinical models to study the pathogenesis and measure the effectiveness of new antigens are limited ${ }^{50}$. The four candidates that reached clinical trials were a therapeutic whole-cell vaccine, a partially autolysed vaccine, a pilus-based vaccine and a PorA-based vaccine, none of which was effective ${ }^{51}$. A recent retrospective case-control study generated new hopes for developing an effective vaccine against $N$. gonorrhoeae. Reduced rates of gonorrhoea were found in individuals at sexual health clinics following vaccination with an OMV-based vaccine (MeNZB) that was developed to control an outbreak of the closely related pathogen Neisseria meningitidis B in New Zealand between 2004 and 2008; the estimated effectiveness of the vaccine against gonorrhoea was $31 \%$ (REF. ${ }^{52}$ ). Although the effectiveness was relatively low, mathematical modelling suggested that a vaccine with moderate protective efficacy might have a significant effect on the burden of gonorrhoea ${ }^{53}$. Other OMV-based vaccines developed for N. meningitidis B showed some levels of efficacy against $N$. gonorrhoeae in specific countries, including Cuba and Canada ${ }^{54}$, which further suggests cross-protection ${ }^{50}$. A clinical study is planned to test the efficacy of a serogroup B meningococcus ( N. meningitidis serogroup $B$ ) vaccine that contains OMVs (4CMenB, brand name Bexsero) in protecting vulnerable populations from infection with N. gonorrhoeae 55 .

Other targets have also been identified and showed protection in preclinical mouse models. For example, a peptide mimetic of a highly bactericidal lipooligosaccharide epitope and a porin B in a viral delivery system demonstrated a reduced duration of infection in mice. Additional antigens expressed as recombinant proteins have shown the ability to induce antibodies with functional activity; they include the nitrite reductase AniA, the transferrin-binding proteins TbpA and TbpB and the methionine uptake receptor MetQ (Supplementary Table 1).

For progression of the development of $N$. gonorrhoeae vaccine candidates, specific challenges need to be taken into consideration. At the preclinical level, new and alternative in vitro, in vivo and ex vivo models that resemble $N$. gonorrhoeae infections need to be developed to properly study the functional nature of antibodies. Human specificity of N. gonorrhoeae limits the development and use of proper animal models that lack several human-specific factors involved in infection (for example, transferrin-binding and lactoferrin-binding proteins, factor $\mathrm{H}, \mathrm{C} 4 \mathrm{~b}$ and cellular receptors). Although a female mouse model of lower genital tract infection is available ${ }^{56}$, it might be improved by the development of transgenic mice strains. In addition, the role of mucosal immunity should be investigated to develop vaccine candidates that can induce the proper functional immune response at the site of infection. At the clinical level, the lack of established correlates of protection may be a challenge to understand what type of immunity has to be induced, and trial design may be problematic for the identification of a suitable target population, especially in the context of sexually transmitted diseases. Human challenge models that involve experimental urethral infection of male volunteers are possible due to low risk of complication, whereas infection of women is ethically prohibited. However due to different pathogenesis in men and women ${ }^{57}$, human challenge models in men may be limited for assessing vaccine development in women. Finally, the duration of vaccine-induced protection and the effect on asymptomatic carriage should also be considered.

Although various challenges to develop an effective vaccine against $N$. gonorrhoeae exist, the reduced rate of gonorrhoea observed after use of the $N$. meningitidis OMV-based vaccine provided evidence that the development of a vaccine is feasible. More studies elucidating the type of immune response that is mediated by the vaccine and the antigens that conferred protection will guide future vaccine discovery and development.

Pseudomonas aeruginosa. Despite substantial efforts, no licensed vaccines against $P$. aeruginos $a$ are available, and no potential candidates are currently in clinical trials ${ }^{58}$.

Vaccine targets for $P$. aeruginosa have been discovered and characterized ${ }^{59}$. The vaccine candidates tested so far in humans consisted of antigens that target single virulence mechanisms, such as the outer membrane proteins OprF and OprI, flagella ${ }^{60}$ and exopolysaccharide alginate $^{61}$ (Supplementary Table 1 ). None of them progressed into late-stage development and even the most promising OprF-OprI fusion protein showed disappointing clinical efficacy results ${ }^{62}$. As $P$. aeruginosa exhibits several virulence mechanisms and adapts to host environments (for example, by establishing biofilms), it is important to consider using multiple vaccine candidates in combination. A recent reverse vaccinology approach identified multiple antigens that, in combination, effectively controlled $P$. aeruginosa infection in a mouse model of acute pneumonia ${ }^{63}$. However, more research to understand the molecular mechanisms of $P$. aeruginosa infection pathogenesis and the relevant effectors at the different stages of infection is required. In addition, in vitro, ex vivo and animal models that resemble key human niches (for example, the lung or urinary tract) and the specific infections caused in the target populations should be developed and tested for robustness and predictability. Target populations may not mount a strong immune response as some individuals are likely to be immunocompromised or elderly. Hence, vaccine research should also consider testing different adjuvants that can improve specific immune responses (BOX 2). Multiple vaccine candidates in combination should then move to early clinical studies in specific target populations, and efficacy studies can inform on future vaccine development.

Klebsiella pneumoniae. Several vaccine targets against K. pneumoniae have been described in the past few decades $^{64}$ (Supplementary Table 1). Different preparations of plain capsule polysaccharide (CPS) vaccines have been tested in preclinical and clinical studies, and used to produce hyperimmune human sera as a therapeutic. Only a few examples of conjugates with CPS are described in the literature, including one example with 
a semisynthetic glycoconjugate approach ${ }^{65,66}$. Recently, bioconjugate vaccines comprising CPS from two $K$. pneumoniae serotypes have been shown to be immunogenic and efficacious, protecting mice against lethal infection ${ }^{67}$. Although all these candidates induced functional antibodies in animal models, it is unlikely that a capsule-based vaccine will be successful considering the presence of 77 CPS serotypes with limited or no cross-reactivity, and a vaccine would have to include at least 24 major serotypes to cover $70 \%$ of K. pneumoniae strains ${ }^{68}$. A different situation is described for the O polysaccharides (OPS) of K. pneumoniae lipopolysaccharides: although eight $\mathrm{O}$ serotypes have been described, epidemiological studies suggest that four OPS would cover approximately $80 \%$ of the clinical isolates worldwide. OPS-based conjugate vaccines have been described. In particular, conjugation of four OPS to $P$. aeruginosa flagellin elicited antibodies that protected mice against $K$. pneumoniae infection ${ }^{69}$, which opens up the possibility to treat common hospital-acquired infections by combining specific antigens. Outer membrane proteins as single recombinant antigens have been investigated as potential vaccine candidates and induce protective immunity in preclinical animal models of infection $^{64}$. More recently it was shown that a vaccine candidate derived from K. pneumoniae OMVs conferred protection in a preclinical animal model, and the mechanism was dependent on both humoral and cellular immunity ${ }^{70}$; this result emphasizes that generalized modules for membrane antigens (GMMA) technology is a promising strategy for vaccine development (BOX 2).

Despite intensive research on K. pneumoniae vaccine antigens at the preclinical level, no vaccines have been approved or have recently progressed into late-stage clinical trials ${ }^{58}$. Promising candidates have been described in preclinical models, but for progression into development, some key challenges have to be considered: reliable in vitro, ex vivo and in vivo animal models are lacking for K. pneumoniae; although it is well recognized that K. pneumoniae is a leading cause of hospital-acquired infections, robust and reliable estimations of the disease burden are currently not fully available, which hampers proper selection of the most appropriate population to target during clinical trials; as K. pneumoniae can colonize the human gut and respiratory tract as a commensal, the implication of asymptomatic carriage should be studied to understand whether K. pneumoniae colonization is directly associated with progression to extraintestinal infection, as has been suggested ${ }^{71}$.

Salmonella enterica subsp. enterica serovars Typhi and Paratyphi A. A vaccine against Salmonella enterica subsp. enterica serovar Typhi will be particularly beneficial for infants and young children in endemic countries, such as countries in South Asia, South-East Asia and sub-Saharan Africa, as well as for travellers ${ }^{72}$. There are 20 marketed vaccines against Salmonella Typhi, and many others are in development (Supplementary Table 1). The marketed vaccines are live attenuated Ty21a oral vaccines and Vi polysaccharide vaccines with intrinsic limitations such as the need for multiple doses and lack of immunological memory, affinity maturation and limited duration of antibody response ${ }^{73}$. To overcome these limitations, novel strategies have been proposed, such as conjugation of $\mathrm{Vi}$ polysaccharide to an appropriate carrier protein, which enables the conversion of the T cell-independent Vi polysaccharide antigen into a T cell-dependent antigen. Although a phase III study of a Salmonella Typhi Vi conjugate vaccine showed more than $90 \%$ efficacy in children $2-5$ years old ${ }^{74}$, the lack of a clear commercial incentive for developing vaccines against Salmonella Typhi slowed down the introduction of the vaccines onto the market. Only recently have $\mathrm{Vi}$ glycoconjugate vaccines have been licensed in India and China ${ }^{73}$, owing to the expanding network of vaccine manufacturers in emerging economies ${ }^{72}$. Incentive for the development of Salmonella Typhi vaccines has also come from global health vaccine institutes, such as the International Vaccine Institute in Seoul, South Korea, and the GSK Vaccines Institute for Global Health (GVGH) in Siena, Italy, as well as key academic institutions such as the US National Institutes of Health and the Center for Vaccine Development and Global Health (CVD) at the University of Maryland, USA ${ }^{75,76}$. Typbar TCV, which is a typhoid conjugate vaccine that consists of the Salmonella Typhi Vi polysaccharide conjugated to tetanus toxoid carrier protein, was recently prequalified by the WHO, and is currently in effectiveness trials in several countries ${ }^{77}$. Many other vaccine candidates are in development (Supplementary Table 1) by different producers and should be supported to guarantee healthy competition and production capacity in the market.

No vaccine is available for Salmonella enterica subsp. enterica serovar Paratyphi A due to lower commercial interest. Few vaccines against Salmonella Paratyphi A are currently in development, those that are in development are primarily based on whole-cell live attenuated strains or on the specific OPS (O:2) conjugated to different carrier proteins ${ }^{78}$, and only one is in a phase I $\operatorname{trial}^{79}$. As Salmonella Paratyphi A has low incidence and low associated mortality and morbidity, uptake of development by manufacturers and by the population of a stand-alone vaccine is unlikely, and major ongoing work focuses on the development of a bivalent vaccine against both Salmonella Typhi and Salmonella Paratyphi A (Supplementary Table 1). GVGH has recently transferred the technology to develop a bivalent glycoconjugate vaccine targeting both Salmonella Typhi and Salmonella Paratyphi A to Biological E (India) ${ }^{80}$.

Invasive non-typhoidal Salmonella. Few candidate vaccines against invasive non-typhoidal Salmonella are currently in preclinical development (Supplementary Table 1). Proof-of-principle studies in animal models have demonstrated efficacy for live attenuated vaccines and subunit vaccines that target the OPS, flagellin proteins and other outer membrane proteins of Salmonella enterica subsp. enterica serovar Typhimurium and Salmonella enterica subsp. enterica serovar Enteritidis ${ }^{81}$. Poor immunogenicity of OPS can be markedly enhanced through chemical linkage to carrier proteins ${ }^{82,83}$. The CVD has developed a bivalent conjugate vaccine with Salmonella Typhimurium and Salmonella Enteritidis OPS covalently linked to the homologous flagellin 
subunits $^{84}$. More recently, a GMMA-based bivalent vaccine has been proposed. GMMA present OPS chains and outer membrane proteins in association with the bacterial membrane, with their native orientation and conformation $^{85}$. Other promising vaccine approaches against invasive non-typhoidal Salmonella include live attenuated candidates, which can be delivered orally and induce robust mucosal and $\mathrm{T}$ cell immunity ${ }^{73}$. The CVD is also developing live attenuated, oral vaccines for both Salmonella Typhimurium and Salmonella Enteritidis ${ }^{81}$.

The limited preclinical activity does not reflect the feasibility to develop an effective vaccine, but rather highlights a clear lack of resources and incentive to drive preclinical development forward. It would be important to better understand the epidemiology and burden of invasive non-typhoidal Salmonella infection at a global and regional level. Combination with a vaccine against Salmonella Typhi could increase commercial attractiveness.

Shigella species. No vaccine is currently widely available against Shigella species, but there is robust global health interest in developing such a vaccine, and the pipeline includes a moderate number of candidates, a few of which are currently in phase I and phase II trials (Supplementary Table 1). A vaccine against Shigella species would likely be offered as a routine childhood vaccination in endemic regions, predominately in LMICs, and as a vaccine for travellers.

As natural immunity against Shigella species is serotype specific, the key target of the vaccine candidates is $\mathrm{OPS}^{86}$. A glycoconjugate based on a synthetic carbohydrate of Shigella flexneri type 2a has recently been shown to be safe and immunogenic in a Phase 1 trial $^{87}$. Phase I trials of monovalent bioconjugates against Shigella $d y s$ enteriae $\mathrm{O} 1$ and $S$. flexneri $2 \mathrm{a}^{88}$ have been completed by LimmaTech Biologics ${ }^{89}$. GVGH has used the GMMA approach to develop a vaccine against Shigella sonnei, which is already being tested in phase I and phase II clinical trials ${ }^{90}$, and is ready for testing a tetravalent vaccine combining S. sonnei to $S$. flexneri GMMA ${ }^{91}$.

Protein-based subunit vaccine candidates are also in development, including the DB Fusion, which is produced by the genetic fusion of the type III secretion system (TTSS) proteins IpaB and $\mathrm{IpaD}^{92}$ and $34-\mathrm{kDa}$ outer membrane protein A (OmpA) from $S$. flexneri $2 a^{93}$. Selective genetic manipulation has been used for the development of orally administered immunogenic live attenuated vaccines (Supplementary Table 1) that did not exhibited any safety concerns ${ }^{86}$.

Clinical development for Shigella should be accelerated, testing promising vaccine candidates in the target population as quickly as possible. Also, considering that the greatest burden of disease is in LMICs, development of combination vaccines, covering other enteric diseases such as enterotoxigenic $E$. coli infection, should be explored.

Group A Streptococcus. Currently, no vaccine is available against group A Streptococcus (GAS). The only vaccines that have been tested in clinical trials are based on the $\mathrm{M}$ protein, the major virulence determinant of the $\operatorname{organism}^{94}$. The M protein is a coiled-coil protein that consists of three domains: an A-repeat amino-terminal domain, which is highly variable and used for molecular typing (emm typing); a B-repeat domain (some antibodies to this region are cross-reactive with host tissue proteins, triggering an inflammatory response leading to permanent heart damage); and a conserved C-repeat domain. A 26-valent amino-terminal M protein-based vaccine (comprising M protein fragments from 26 different serotypes of GAS) was shown to be safe and immunogenic in a phase I and phase II clinical trial in human adult volunteers ${ }^{95}$. The 26 -valent vaccine was reformulated into a 30 -valent vaccine to increase serotype coverage ${ }^{96}$, including certain serotypes that circulate in low-income countries ${ }^{97}$. Vaccines based on the conserved carboxy-terminal part of $\mathrm{M}$ protein (the J8 and J14 vaccines and the StreptInCor vaccine $)^{98,99}$ could provide broader immunity than typeserotype-specific vaccines, but clinical trials now need to determine whether they are sufficiently immunogenic and protective in humans ${ }^{100}$. Combining the synthetic peptide-based J8 vaccine with an inactive form of the streptococcal CXC chemokine protease protected mice against both intraperitoneal challenge and skin infection in a novel pyoderma mouse model ${ }^{101}$. Other vaccines are based on conserved protein antigens (for example, streptococcal C5a peptidase, streptolysin O (Slo) and interleukin-8 (IL-8) protease (SpyCEP)) and are in development, but none of them has entered clinical trials yet ${ }^{102,103}$. GAS clinical isolates express group A carbohydrate, a conserved surface polysaccharide, which has also been proposed for the design of an efficacious anti-GAS glycoconjugate vaccine ${ }^{104,105}$ (Supplementary Table 1).

GAS vaccines have been described as impeded

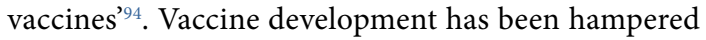
by the need for better epidemiological data in most developing countries, by lack of data regarding strain diversity, by lack of surrogate markers for immune protection in humans and lack of robust animal models, and by perceived safety concerns ${ }^{94}$. The concerns are based on the theoretical risk that the vaccines elicit an autoimmune reaction leading to the development of acute rheumatic fever, although all contemporary GAS vaccines under development have been designed to negate this risk ${ }^{100}$. Perhaps one of the most substantial obstacles in GAS vaccine development is the fact that the severest GAS diseases primarily affect $\mathrm{LMICs}^{100}$ and commercial incentive is low. Furthermore, there is not a clear path for clinical trial design that would lead to GAS vaccine registration; the relatively low incidence of GAS-induced acute rheumatic fever, rheumatic heart disease and invasive disease as well as the time delay between initial infection and disease makes these diseases potentially difficult end points for phase III efficacy studies. A vaccine-induced protective effect against pharyngitis (the most frequent symptomatic GAS infection) would instead provide a proof of concept for efficacy, to be followed by studies for other clinical syndromes, with the goal being the availability of a preventive vaccine for the most prevalent GAS-associated diseases globally ${ }^{106}$. 
Mycobacterium tuberculosis. The Mycobacterium bovis bacille Calmette-Guérin (BCG) vaccine, composed of an attenuated strain of $M$. bovis, is the only licensed and widely used vaccine for Mycobacterium tuberculosis. Global vaccine coverage is estimated at $\sim 90 \%$ (REF. ${ }^{107}$ ), but the efficacy of the vaccine is highly variable, ranging from substantial protection shown in a trial conducted in the UK by the Medical Research Council to the absence of a clinical benefit in a trial performed in southern India ${ }^{108}$. Despite the observed great variation of BCG efficacy, tuberculosis vaccine research and development has been underfunded, especially considering the public health and socio-economic burden of the disease, and progress was slow until the recent publication of two positive efficacy trials. The first showed that revaccination of adolescents who are at high risk of infection provided a protective effect (in $45.4 \%$ of the individuals) against $M$. tuberculosis ${ }^{109}$. The second trial demonstrated that vaccination of adults infected with $M$. tuberculosis with the adjuvanted recombinant peptide vaccine M72/AS01 provided 50\% protection against progression to pulmonary tuberculosis for at least 3 years ${ }^{110,111}$. Both vaccines were shown to be safe in the populations tested. The pipeline of products in clinical development is diverse, with various live attenuated or inactivated mycobacterium-derived candidates (VPM1002, MTBVAC and DAR-901), adjuvanted recombinant proteins (H56:IC31, H4-IC31, ID93/GLA-SE and M72/AS01E) and recombinant viral vectors (MVA85A, Ad5Ag85A, ChAdOx185A and TB/FLU-04L), and the candidates are progressing through human evaluation ${ }^{112}$ (Supplementary Table 1).

Going forward, more studies are needed to fully understand the mechanisms of immune protection and pathogen biology. Clinical trials are challenging to design and to conduct due to the lack of reliable correlates of immune protection or biomarkers, the difficulty of controlled human infection studies and difficult trial infrastructure in rural areas.

\section{Conclusions and outlook}

AMR is an urgent global health threat ${ }^{4}$, and the development of vaccines against key pathogens with a complex resistance profile and high incidence of severe infections may be a promising solution. The problem of drug-resistant infection is often thought to be restricted mainly to the hospital setting, where severe infections due to antimicrobial-resistant pathogens occur. However, resistant strains often emerge in the community, where they spread and enter the hospital setting ${ }^{16,17}$. Although not all the vaccines can eradicate the target pathogens, a reduction in the incidence of infection can reduce the use of antibiotics and therefore the emergence of AMR (direct effect). Importantly, not only vaccines against bacteria contribute to a reduction in the consumption of antibiotics but so do vaccines against viruses, owing to a decrease in inappropriate antibiotic prescriptions (indirect effect). Vaccines are also important in the veterinary and agricultural settings, where antibiotics are overused.

AMR seems to be perceived as an urgent medical need in HICs but not so much in LMICs ${ }^{18,113}$. However, the prevalence of antimicrobial-resistant pathogens is high in countries of all income levels, and there is substantial overlap for resistant species, including K. pneumoniae, $P$. aeruginosa and $S$. aureus ${ }^{20}$. Some antimicrobialresistant pathogens, for example, Salmonella and Shigella species, are prevalent mainly in LMICs, and understanding the relative 'value' of vaccines against such pathogens is becoming increasingly crucial to inform priority setting for investment and introduction decisions, and to increase the probability that safe and effective vaccines will be developed. Furthermore, combination vaccines, covering multiple diseases, can enhance commercial attractiveness and accelerate vaccine development especially for LMICs.

To maximize the impact of vaccines in reducing the emergence of AMR, most of the population that is at risk of infection should be vaccinated and in all countries where the antimicrobial-resistant pathogens are endemic. Unfortunately, for most of the key antimicrobial-resistant pathogens, vaccines are not yet available. Several candidate vaccines are in different phases of development (FIG. 3); most are still in preclinical testing and just a few are in clinical development (Supplementary Table 1). Clinical development of candidate vaccines for $S$. aureus, C. difficile, P. aeruginosa, ExPEC and N. gonorrhoeae has so far failed. This is in part due to the complexity of the pathogens and in part due to the difficulty of conducting efficacy trials. Research on the pathogenesis and host immune responses will provide much needed information to guide vaccine design. Compared with a few years ago, we now have a much better understanding of the contribution of functional antibodies, cell-mediated immunity and innate immunity in mediating protection against these pathogens. This information is being used as end points in preclinical research to select candidate vaccines and in clinical studies to confirm the data obtained in human trials.

Novel approaches are being explored for the development of vaccines against antimicrobial-resistant pathogens (BOX 2): OMV-based vaccines are at the preclinical stage for many pathogens, and innovative synthetic and bioconjugation strategies are replacing more traditional conjugation approaches and are more advanced in terms of clinical development (for example, for Shigella species or ExPEC). New adjuvants may increase vaccine efficacy, especially of protein-based vaccines ${ }^{9}$. Older technologies, such as live attenuated and inactivated vaccines, due to their simplicity and low cost of manufacture, remain a good alternative. In addition, more traditional approaches can be improved (that is, the design of safer live attenuated vaccines ${ }^{114}$, simplifying processes for polysaccharide purification and improving production of glycoconjugates) $)^{115}$.

It takes a long time to develop a new vaccine, usually between 10 and 20 years. To respond to the AMR crisis, pharmaceutical companies should transform the vaccine development process, which might entail new technologies and new vaccine platforms, and it would be also fundamental to accelerate clinical studies and to change the interactions with regulatory authorities. In this context, continuous discussions with regulatory and health authorities on the requirements for AMR vaccines 


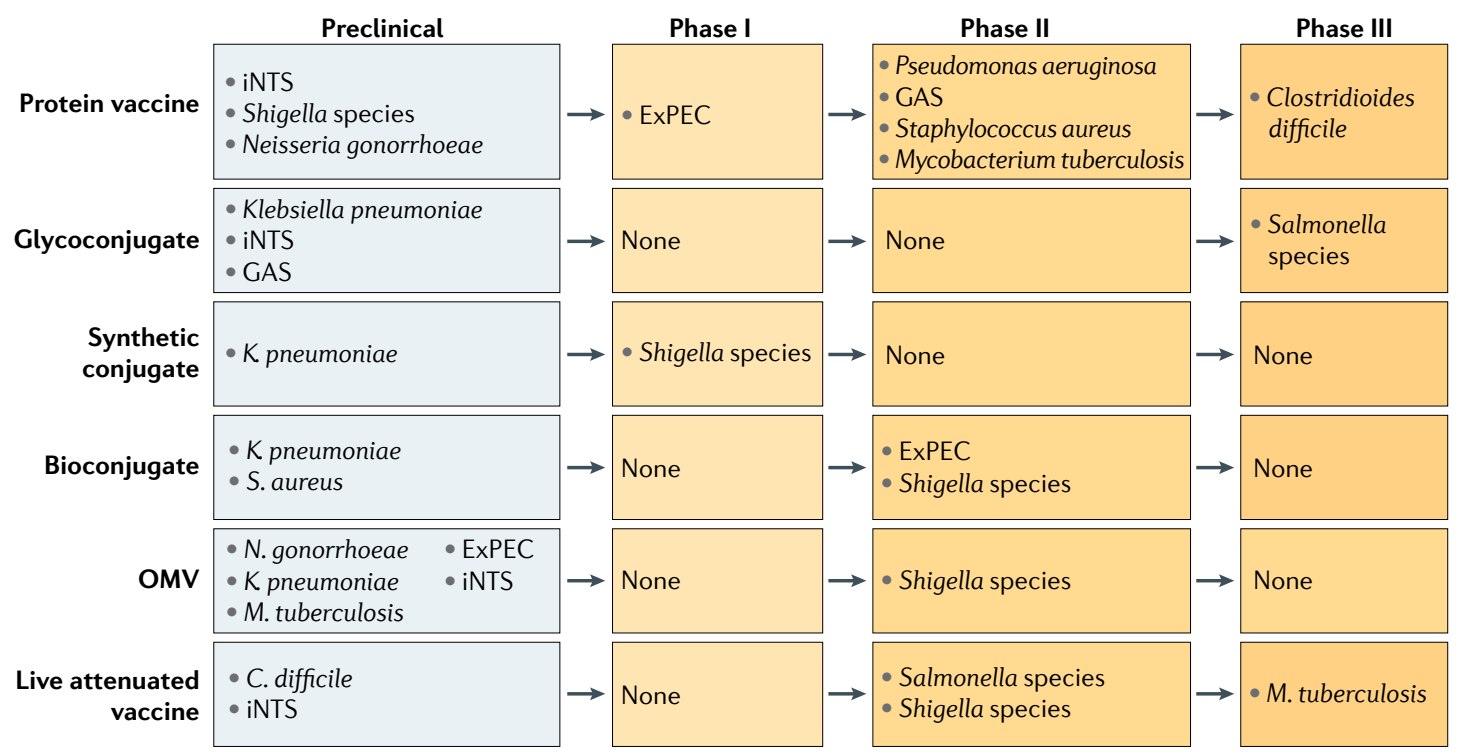

Fig. 3 | Vaccine development for antimicrobial-resistant pathogens. Shown are vaccine candidates that are currently at different stages of development. Various vaccine technologies and platforms (protein vaccine, glycoconjugate, synthetic conjugate, bioconjugate, outer membrane vesicles (OMVs) and live attenuated vaccines) are being applied to identify and develop such vaccines, as indicated. (see also Supplementary Table 1). ExPEC, extraintestinal pathogenic Escherichia coli; GAS, group A Streptococcus; iNTS, invasive non-typhoidal Salmonella.

are crucial to identify alternative ways to test novel vaccines in specific clinical trials to accelerate the overall process. For example, testing novel vaccine candidates directly in the target population could accelerate vaccine development. For LMICs, it would be constructive to enlist a contract manufacturer at an early stage of the process and to strengthen international collaborations and public-private partnerships, including funders and policymakers. The fast development of candidate vaccines against severe acute respiratory syndrome coronavirus 2 (SARS-CoV-2) may provide important guidance to accelerate the process for antimicrobial-resistant pathogens ${ }^{116}$.

Despite the general agreement that vaccines have great potential in reducing AMR, and the accumulating evidence corroborating the impact of existing vaccines on reducing the emergence and spread of AMR, predicting the potential impact of vaccines that are currently under development is challenging. This is due to the difficulty in recognizing all the various parameters that influence the expansion of AMR, and because data on antibiotic use for different infections are still fragmented and difficult to retrieve. Currently, cost-effectiveness analyses performed for future vaccines do not usually consider their impact on AMR, which substantially decreases their estimated value. Hence, different and more sophisticated methods to measure cost-effectiveness need to be implemented ${ }^{117,118}$.

Other tools such as monoclonal antibodies, bacteriophages, microbiota targeting and innovative diagnostic tools are emerging strategies that can complement vaccines in the fight against $\mathrm{AMR}^{119}$. There is not a single solution, and a globally integrated strategy is required to combat AMR effectively.

Published online 4 February 2021
1. Kapoor, G., Saigal, S. \& Elongavan, A. Action and resistance mechanisms of antibiotics: a guide for clinicians. J. Anaesthesiol. Clin. Pharmacol. 33 300-305 (2017).

2. Mladenovic-Antic, S. et al. Correlation between antimicrobial consumption and antimicrobial resistance of Pseudomonas aeruginosa in a hospital setting: a 10-year study. J. Clin. Pharm. Ther. 41, 532-537 (2016).

3. Hay, S. I. et al. Measuring and mapping the global burden of antimicrobial resistance. BMC Med. 16 78 (2018).

4. IACG No Time To Wait: Securing The Future From Drug-Resistant Infections (2019).

5. United Nations. Political Declaration of the High-Leve Meeting of the General Assembly on Antimicrobial Resistance, A/71/L.2. 22 (2016).

6. Aslam, B. et al. Antibiotic resistance: a rundown of a global crisis. Infect. Drug Resist. 11, 1645-1658 (2018).

7. Rappuoli, R., Santoni, A. \& Mantovani, A. Vaccines: an achievement of civilization, a human right, our health insurance for the future. J. Exp. Med. 216 7-9 (2019).
8. Kennedy, D. A. \& Read, A. F. Why the evolution of vaccine resistance is less of a concern than the evolution of drug resistance. Proc. Natl Acad. Sci. USA 115, 12878-12886 (2018).

This review compares emergence of drug resistance and vaccine resistance and explains why vaccine resistance is not a major concern.

9. Baker, S. J., Payne, D. J., Rappuoli, R. \& De Gregorio, E. Technologies to address antimicrobial resistance. Proc. Natl Acad. Sci. USA 115, 12887-12895 (2018). This review describes how innovative vaccine technologies have the potential to boost the development of vaccines targeting several classes of multidrug-resistant bacteria.

10. Klugman, K. P. \& Black, S. Impact of existing vaccines in reducing antibiotic resistance: Primary and secondary effects. Proc. Natl Acad. Sci. USA 115 12896-12901 (2018).

11. Jansen, K. U., Knirsch, C. \& Anderson, A. S. The role of vaccines in preventing bacterial antimicrobial resistance. Nat. Med. 24, 10-19 (2018). This review highlights the value of vaccines as one of the modalities to combat AMR globally, using select examples.
12. Cassini, A. et al. Attributable deaths and disabilityadjusted life-years caused by infections with antibiotic-resistant bacteria in the EU and the European Economic Area in 2015: a population-level modelling analysis. Lancet Infect. Dis. 19, 56-66 (2019).

13. CDC. Antibiotic Resistance Threats in the United States, 2019. https://www.cdc.gov/DrugResistance/ Biggest-Threats.html (Department of Health and Human Services, 2019).

This is a CDC report with the latest national death and infection estimates that underscore the continued threat of antibiotic resistance in the USA.

14. World Health Organization. Global priority list of antibiotic-resistant bacteria to guide research, discovery, and development of new antibiotics. https://www.who.int/medicines/publications/ WHO-PPL-Short_Summary_25Feb-ET_NM_WHO. pdf?ua=1 (2017)

This WHO document reports a list of antibioticresistant bacteria to help in prioritizing research and development investments for new and effective interventions 
15. CDC, Healthcare-associated infections, https:// www.cdc.gov/hai/patientsafety/ar-hospitals.htm

16. Hersh, A. L., Chambers, H. F., Maselli, J. H. \& Gonzales, R. National trends in ambulatory visits and antibiotic prescribing for skin and soft-tissue infections. Arch. Intern. Med. 168, 1585-1591 (2008).

17. Prosperi, M. et al. Molecular epidemiology of community-associated methicillin-resistant Staphylococcus aureus in the genomic era: a cross-sectional study. Sci. Rep. 3, 1902 (2013)

18. Khan, M. S. et al. 'LMICs as reservoirs of AMR'. a comparative analysis of policy discourse on antimicrobial resistance with reference to Pakistan. Health Policy Plan. 34, 178-187 (2019).

19. FAO Global Forum on Food Security and Nutrition http://www.fao.org/3/cb0863en/cb0863en.pdf

20. Founou, R. C., Founou, L. L. \& Essack, S. Y. Clinical and economic impact of antibiotic resistance in developing countries: a systematic review and meta-analysis. PLoS ONE 12, e0189621 (2017). This study analyses the published literature on the clinical and economic implications of AMR in developing countries.

21. Ayukekbong, J. A., Ntemgwa, M. \& Atabe, A. N The threat of antimicrobial resistance in developing countries: causes and control strategies. Antimicrob. Resist. Infect. Control. 6, 47 (2017).

22. Bagnoli, F. \& Payne, D. J. Reaction: alternative modalities to address antibiotic-resistant pathogens Chem 3, 369-372 (2017).

23. Romanò, L. et al. Hepatitis B vaccination. Hum. Vaccin. Immunother. 11, 53-57 (2015)

24. Zanetti, A. R., Van Damme, P. \& Shouval, D. The global impact of vaccination against hepatitis B: a historical overview. Vaccine 26, 6266-6273 (2008).

25. Octavia, S. et al. Newly emerging clones of Bordetella pertussis carrying prn2 and ptxP3 alleles implicated in Australian pertussis epidemic in 2008-2010. J. Infect. Dis. 205, 1220-1224 (2012).

26. Cherry, J. D. Epidemic pertussis in 2012-the resurgence of a vaccine-preventable disease. N. Engl. J. Med. 367, 785-787 (2012).

27. Mooi, F. R. et al. Polymorphism in the Bordetella pertussis virulence factors P.69/pertactin and pertussis toxin in the Netherlands: temporal trends and evidence for vaccine-driven evolution. Infect. Immun. $\mathbf{6 6}$ 670-675 (1998)

28. Mishra, R. P., Oviedo-Orta, E., Prachi, P., Rappuoli, R. $\&$ Bagnoli, F. Vaccines and antibiotic resistance. Curr. Opin. Microbiol. 15, 596-602 (2012).

29. Miller, E., Andrews, N. J., Waight, P. A., Slack, M. P. $\&$ George, R. C. Herd immunity and serotype replacement 4 years after seven-valent pneumococcal conjugate vaccination in England and Wales: an observational cohort study. Lancet Infect. Dis. 11 760-768 (2011)

30. Moore, M. R. et al. Population snapshot of emergent Streptococcus pneumoniae serotype 19A in the United States, 2005. J. Infect. Dis. 197, 1016-1027 (2008).

31. Lipsitch, M. \& Siber, G. R. How can vaccines contribute to solving the antimicrobial resistance problem? $\mathrm{mBiO}$ 7, e00428-16 (2016).

\section{This minireview describes the significant} contributions of current vaccines and the potential of future vaccines in controlling AMR and elucidates the mechanisms by which this can occur.

32. Sihvonen, R., Siira, L., Toropainen, M., Kuusela, P. \& Patari-Sampo, A. Streptococcus pneumoniae antimicrobial resistance decreased in the Helsinki Metropolitan Area after routine 10-valent pneumococcal conjugate vaccination of infants in Finland. Eur. J. Clin. Microbiol. Infect. Dis. 36, 2109-2116 (2017)

33. John, T. J., Cherian, T. \& Raghupathy, P. Haemophilus influenzae disease in children in India: a hospital perspective. Pediatr. Infect. Dis. J. 17, S169-S171 (1998).

34. Oliver, S. P., Murinda, S. E. \& Jayarao, B. M. Impact of antibiotic use in adult dairy cows on antimicrobial resistance of veterinary and human pathogens: a comprehensive review. Foodborne Pathog. Dis. 8 , 337-355 (2011).

35. No authors. Standing up to antimicrobial resistance Nat Rev Microbiol 8, 836 (2010)

36. McEwen, S. A. \& Fedorka-Cray, P. J. Antimicrobial use and resistance in animals. Clin. Infect. Dis. 34 (Suppl. 3), S93-S106 (2002).

37. Hoelzer, K. et al. Vaccines as alternatives to antibiotics for food producing animals. Part 1 : challenges and needs. Vet. Res. 49, 64 (2018)
38. Tekle, Y. I. et al. Controlling antimicrobial resistance through targeted, vaccine-induced replacement of strains. PLOS ONE 7, e50688 (2012).

39. US National Library of Medicine. ClinicalTrials.gov https://clinicaltrials.gov/ct2/show/NCT01887912 (2013).

40. US National Library of Medicine. ClinicalTrials.gov https://clinicaltrials.gov/ct2/show/NCT03090191? term=vaccine $\&$ cond=clostridium +difficile $\&$ rank $=7$ (2017).

41. US National Library of Medicine. ClinicalTrials.gov https://clinicaltrials.gov/ct2/show/NCT02316470? term=vaccine (2014).

42. US National Library of Medicine. ClinicalTrials.gov https://clinicaltrials.gov/ct2/show/NCT04026009 (2019).

43. Pechine, S., Bruxelle, J. F., Janoir, C. \& Collignon, A Targeting clostridium difficile surface components to develop immunotherapeutic strategies against Clostridium difficile infection. Front. Microbiol. 9 , 1009 (2018)

44. Starks, C. M. et al. Optimization and qualification of an assay that demonstrates that a FimH vaccine induces functional antibody responses in women with histories of urinary tract infections. Hum. Vaccin. Immunother. https://doi.org/10.1080/21645515.202 0.1770034 (2020)

45. Huttner, A. \& Gambillara, V. The development and early clinical testing of the ExPEC4V conjugate vaccine against uropathogenic Escherichia coli. Clin. Microbiol. Infect. 24, 1046-1050 (2018)

46. Huttner, A et al. Safety, immunogenicity, and preliminary clinical efficacy of a vaccine against extraintestinal pathogenic Escherichia coli in women with a history of recurrent urinary tract infection: a randomised, single-blind, placebo-controlled phase $1 \mathrm{~b}$ trial. Lancet Infect. Dis. 17, 528-537 (2017).

47. Fattom, A et al. Efficacy profile of a bivalent Staphylococcus aureus glycoconjugated vaccine in adults on hemodialysis: phase III randomized study. Hum. Vaccin. Immunother. 11, 632-641 (2015).

48. Fowler, V. G. et al. Effect of an investigational vaccine for preventing Staphylococcus aureus infections after cardiothoracic surgery: a randomized trial. JAMA 309 1368-1378 (2013).

49. Pozzi, C. et al. Vaccines for Staphylococcus aureus and target populations. Curr. Top. Microbiol. Immunol. 409, 491-528 (2017).

50. Edwards, J. L., Jennings, M. P. \& Seib, K. L. Neisseria gonorrhoeae vaccine development: hope on the horizon? Curr. Opin. Infect. Dis. 31, 246-250 (2018).

51. Vincent, L. R. \& Jerse, A. E. Biological feasibility and importance of a gonorrhea vaccine for global public health. Vaccine 37, 7419-7426 (2018)

52. Petousis-Harris, H. et al. Effectiveness of a group B outer membrane vesicle meningococcal vaccine against gonorrhoea in New Zealand: a retrospective case-control study. Lancet 390, 1603-1610 (2017)

53. Craig, A. P. et al. The potential impact of vaccination on the prevalence of gonorrhea. Vaccine 33 , 4520-4525 (2015).

54. Petousis-Harris, H. \& Radcliff, F. J. Exploitation of Neisseria meningitidis group B OMV vaccines against $\mathrm{N}$. gonorrhoeae to inform the development and deployment of effective gonorrhea vaccines. Front. Immunol. 10, 683 (2019).

55. University of Alabama at Birmingham NIH study to explore vaccine for gonorrhea prevention. https:// www.uab.edu/news/health/item/10852-nih-study-toexplore-vaccine-for-gonorrhea-prevention (2019).

56. Raterman, E. L. \& Jerse, A. E. Female mouse mode of Neisseria gonorrhoeae infection. Methods Mol. Biol. 1997, 413-429 (2019).

57. Edwards, J. L. \& Apicella, M. A. The molecular mechanisms used by Neisseria gonorrhoeae to initiate infection differ between men and women Clin. Microbiol. Rev. 17, 965-981 (2004).

58. The Boston Consulting Group. Vaccines to tackle drug resistant infections. An evaluation of $R \& D$ opportunities (2018).

This report evaluates the development potential of vaccines against pathogens with high levels of AMR

59. Priebe, G. P \& Goldberg J. B. Vaccines for Pseudomonas aeruginosa: a long and winding road. Expert Rev. Vaccines 13, 507-519 (2014).

60. Doring, G., Meisner, C. \& Stern, M. A double-blind randomized placebo-controlled phase III study of a Pseudomonas aeruginosa flagella vaccine in cystic fibrosis patients. Proc. Natl Acad. Sci. USA 104 11020-11025 (2007)

61. Pier, G. B. et al. Human immune response to Pseudomonas aeruginosa mucoid exopolysaccharide (alginate) vaccine. Infect. Immun. 62, 3972-3979 (1994).

62. Rello, J. et al. A randomized placebo-controlled phase II study of a Pseudomonas vaccine in ventilated ICU patients. Crit. Care 21, 22 (2017)

63. Bianconi, I. et al. Genome-based approach delivers vaccine candidates against Pseudomonas aeruginosa. Front. Immunol. 9, 3021 (2018).

64. Choi, M., Tennant, S. M., Simon, R. \& Cross, A. S Progress towards the development of Klebsiella vaccines. Expert Rev. Vaccines 18, 681-691 (2019).

65. Seeberger, P. H. et al. A semi-synthetic glycoconjugate vaccine candidate for carbapenem-resistant Klebsiella pneumoniae. Angew. Chem. Int. Ed. 56 13973-13978 (2017).

66. Zigterman, J. W. et al. Immunogenic properties of octasaccharide-protein conjugates derived from Klebsiella serotype 11 capsular polysaccharide. Infect. Immun. 47, 421-428 (1985).

67. Feldman, M. F. et al. A promising bioconjugate vaccine against hypervirulent Klebsiella pneumoniae. Proc. Natl Acad. Sci. USA 116, 18655-18663 (2019).

68. Follador, R. et al. The diversity of Klebsiella pneumoniae surface polysaccharides. Microb. Genom 2, e000073 (2016)

69. Hegerle, N. et al. Development of a broad spectrum glycoconjugate vaccine to prevent wound and disseminated infections with Klebsiella pneumoniae and Pseudomonas aeruginosa. PLOS ONE 13, e0203143 (2018).

70. Lee, W. H. et al Vaccination with Klebsiella pneumoniae-derived extracellular vesicles protects against bacteria-induced lethality via both humoral and cellular immunity. Exp. Mol. Med. 47, e183 (2015).

71. Martin, R. M. et al. Molecular epidemiology of colonizing and infecting isolates of Klebsiella pneumoniae. $m$ Sphere https://doi.org/10.1128 mSphere.00261-16 (2016).

72. World Health Organization. Typhoid vaccines: WHO position paper, March 2018 - recommendations. Vaccine 37, 214-216 (2019).

73. MacLennan, C. A., Martin, L. B. \& Micoli, F. Vaccines against invasive Salmonella disease: current status and future directions. Hum. Vaccin. Immunother. 10 , 1478-1493 (2014).

74. Lin, F. Y. et al. The efficacy of a Salmonella typhi Vi conjugate vaccine in two-to-five-year-old children N. Engl. J. Med. 344, 1263-1269 (2001)

75. Wahid, R., Salerno-Goncalves, R., Tacket, C. O., Levine, M. M. \& Sztein, M. B. Cell-mediated immune responses in humans after immunization with one or two doses of oral live attenuated typhoid vaccine CVD 909. Vaccine 25, 1416-1425 (2007).

76. Wahid, R. et al. Oral priming with Salmonella Typhi vaccine strain CVD 909 followed by parenteral boost with the $\mathrm{S}$. Typhi Vi capsular polysaccharide vaccine induces CD27 $7^{+}$IgD S. Typhi-specific IgA and IgC B memory cells in humans. Clin. Immunol. 138 187-200 (2011)

77. Jin, C. et al. Efficacy and immunogenicity of a Vi-tetanus toxoid conjugate vaccine in the prevention of typhoid fever using a controlled human infection model of Salmonella Typhi: a randomised controlled, phase 2b trial. Lancet 390, 2472-2480 (2017).

78. Martin, L. B. et al. Status of paratyphoid fever vaccine research and development. Vaccine 34, 2900-2902 (2016).

79. Gat, O. et al. Cell-associated flagella enhance the protection conferred by mucosally-administered attenuated Salmonella Paratyphi A vaccines. PLoS Negl. Trop. Dis. 5, e1373 (2011).

80. Micoli, F. et al. O:2-CRM(197) conjugates against Salmonella Paratyphi A. PLOS ONE 7, e47039 (2012).

81. Tennant, S. M., MacLennan, C. A., Simon, R. Martin, L. B. \& Khan, M. I. Nontyphoidal salmonella disease: current status of vaccine research and development. Vaccine 34, 2907-2910 (2016)

82. Lanzilao, L. et al. Strain selection for generation of O-antigen-based glycoconjugate vaccines against invasive nontyphoidal salmonella disease. PLOS ONE 10, e0139847 (2015)

83. Watson, D. C., Robbins, J. B. \& Szu, S. C. Protection of mice against Salmonella typhimurium with an O-specific polysaccharide-protein conjugate vaccine. Infect. Immun 60, 4679-4686 (1992).

84. Simon, R. et al. Sustained protection in mice immunized with fractional doses of Salmonella Enteritidis core and $O$ polysaccharide-flagellin glycoconjugates. PLoS ONE 8, e64680 (2013). 
85. Micoli, F. et al. Comparative immunogenicity and efficacy of equivalent outer membrane vesicle and glycoconjugate vaccines against nontyphoidal Salmonella. Proc. Natl Acad. Sci. USA 115 10428-10433 (2018).

86. Mani, S., Wierzba, T. \& Walker, R. I. Status of vaccin research and development for Shigella. Vaccine 34 2887-2894 (2016).

87. van der Put, R. M. et al. A synthetic carbohydrate conjugate vaccine candidate against shigellosis: improved bioconjugation and impact of alum on immunogenicity. Bioconjug. Chem. 27, 883-892 (2016).

88. Hatz, C. F. et al. Safety and immunogenicity of a candidate bioconjugate vaccine against Shigella dysenteriae type 1 administered to healthy adults: a single blind, partially randomized phase I study. Vaccine 33, 4594-4601 (2015).

89. Riddle, M. S. et al. Safety and immunogenicity of a candidate bioconjugate vaccine against Shigella flexneri $2 \mathrm{a}$ administered to healthy adults: a singleblind, randomized phase I study. Clin. Vaccine Immunol. 23, 908-917 (2016).

90. Obiero, C. W. et al. A phase 2 a randomized study to evaluate the safety and immunogenicity of the 1790GAHB generalized modules for membrane antigen vaccine against shigella sonnei administered intramuscularly to adults from a shigellosis-endemic country. Front. Immunol. 8, 1884 (2017)

91. Micoli, F. \& MacLennan, C. A. Outer membrane vesicle vaccines. Semin. Immunol. 25, 87-88 (2020).

92. Martinez-Becerra, F. J. et al. Broadly protective Shigella vaccine based on type III secretion apparatus proteins. Infect. Immun. 80, 1222-1231 (2012).

93. Pore, D. \& Chakrabarti, M. K. Outer membrane protein $\mathrm{A}(\mathrm{OmpA})$ from Shigella flexneri $2 \mathrm{a}$ : a promising subunit vaccine candidate. Vaccine 31, 3644-3650 (2013)

94. Steer, A. C. et al. Status of research and development of vaccines for Streptococcus pyogenes. Vaccine 34 2953-2958 (2016)

95. McNeil, S. A et al. Safety and immunogenicity of 26-valent group A Streptococcus vaccine in healthy adult volunteers. Clin. Infect. Dis. 41, 1114-1122 (2005).

96. Dale, J. B., Penfound, T. A., Chiang, E. Y. \& Walton, W. J. New 30-valent M protein-based vaccine evokes cross-opsonic antibodies against non-vaccine serotypes of group A streptococci. Vaccine 29, 8175-8178 (2011)

97. Steer, A. C., Law, I., Matatolu, L., Beall, B. W. \& Carapetis, J. R. Global emm type distribution of group A streptococci: systematic review and implications for vaccine development. Lancet Infect. Dis. 9, 611-616 (2009).

98. Batzloff, M. R. et al. Protection against group A streptococcus by immunization with J8-diphtheria toxoid: contribution of J8- and diphtheria toxoidspecific antibodies to protection. J. Infect. Dis. 187 1598-1608 (2003)

99. Guilherme, L. et al. Towards a vaccine against rheumatic fever. Clin. Dev. Immunol. 13, 125-132 (2006).

100. Sheel, M., Moreland, N. J., Fraser, J. D. \& Carapetis, J. Development of group A streptococcal vaccines: an unmet global health need. Expert Rev. Vaccines 15, 227-238 (2016)

101. Pandey, M. et al. A synthetic M protein peptide synergizes with a CXC chemokine protease to induce vaccine-mediated protection against virulent streptococcal pyoderma and bacteremia. J. Immunol. 194, 5915-5925 (2015).

102. Bensi, G. et al. Multi high-throughput approach for highly selective identification of vaccine candidates: the group A Streptococcus case. Mol Cell Proteomics 11, M111.015693 (2012)

103. Rivera-Hernandez, T. et al. An experimental group a streptococcus vaccine that reduces pharyngitis and tonsillitis in a nonhuman primate model. $\mathrm{mBio}$ https://doi.org/10.1128/mBio.00693-19 (2019).

104. Kabanova, A. et al. Evaluation of a group A Streptococcus synthetic oligosaccharide as vaccine candidate. Vaccine 29, 104-114 (2010)

105. Henningham, A. et al. Virulence role of the GlcNAc side chain of the Lancefield cell wall carbohydrate antigen in non-M1-serotype group A Streptococcus. mBio https://doi.org/10.1128/mBio.02294-17 (2018)

106. Schodel, F. et al. Clinical development strategy for a candidate group A streptococcal vaccine. Vaccine 35 , 2007-2014 (2017)

107. Gamez-Gonzalez, L. B., Hamada, H., Llamas-Guillen, B. A., Ruiz-Fernandez, M. \&
Yamazaki-Nakashimada, M. BCG and Kawasaki disease in Mexico and Japan. Hum. Vaccin. Immunother. 13, 1091-1093 (2017)

108. Mangtani, P. et al. Protection by BCG vaccine against tuberculosis: a systematic review of randomized controlled trials. Clin. Infect. Dis. 58, 470-480 (2014).

109. Nemes, E. et al. Prevention of M. tuberculosis infection with $\mathrm{H} 4: \mathrm{IC} 31$ vaccine or BCG revaccination. N. Engl. J. Med. 379, 138-149 (2018)

110. Tait, D. R. et al. Final analysis of a trial of M72/AS01E vaccine to prevent tuberculosis. N. Engl. J. Med. 381 2429-2439 (2019).

111. Van Der Meeren, O. et al. Phase $2 \mathrm{~b}$ controlled trial of M72/AS01E vaccine to prevent tuberculosis. N. Engl. J. Med. 379, 1621-1634 (2018)

112. Vekemans, J., O'Brien, K. L. \& Farrar, J. Tuberculosis vaccines: rising opportunities. PLOS Med. 16 e1002791 (2019).

113. FAO Global Forum on Food Security and Nutrition, http://www.fao.org/3/cb0863en/cb0863en.pdf

114. Cabral, M. P. et al. Design of live attenuated bacterial vaccines based on D-glutamate auxotrophy. Nat. Commun. 8, 15480 (2017).

115. Micoli, F. et al. Glycoconjugate vaccines: current approaches towards faster vaccine design. Expert Rev. Vaccines 18, 881-895 (2019).

116. Krammer, F. SARS-CoV-2 vaccines in development. Nature 586, 516-527 (2020)

117. Sevilla, J. P., Bloom, D. E., Cadarette, D., Jit, M. $\&$ Lipsitch, M. Toward economic evaluation of the value of vaccines and other health technologies in addressing AMR. Proc. Natl Acad. Sci. USA 115 12911-12919 (2018).

118. Roope, L. S. J. et al. The challenge of antimicrobial resistance: what economics can contribute. Science 364, eaau4679 (2019).

This article highlights prospects for future research on the economics of AMR.

119. Bloom, D. E., Black, S., Salisbury, D. \& Rappuoli, R. Antimicrobial resistance and the role of vaccines. Proc. Natl Acad. Sci. USA 115, 12868-12871 (2018) This article highlights that an integrated strategy that includes antibiotics, vaccines, diagnostic tools, antibodies and new tools targeting the host or the microbiome or delivered by bacteriophages is required to fight AMR effectively.

120. Balsells, E. et al. Infection prevention and control of Clostridium difficile: a global review of guidelines, strategies, and recommendations. J. Glob. Health 6 020410 (2016).

121. Peng, Z. et al. Update on antimicrobial resistance in clostridium difficile: resistance mechanisms and antimicrobial susceptibility testing. J. Clin. Microbiol. 55, 1998-2008 (2017)

122. Asadi Karam, M. R., Habibi, M. \& Bouzari, S. Urinary tract infection: pathogenicity, antibiotic resistance and development of effective vaccines against uropathogenic Escherichia coli. Mol. Immunol. 108, 56-67 (2019).

123. Kourtis, A. P. et al. Vital signs: epidemiology and recent trends in methicillin-resistant and in methicillin-susceptible Staphylococcus aureus bloodstream infections - United States. MMWR Morb Mortal. Wkly. Rep. 68, 214-219 (2019).

124. Unemo, M., Del Rio, C. \& Shafer, W. M. Antimicrobial resistance expressed by Neisseria gonorrhoeae: a major global public health problem in the $21 \mathrm{st}$ century. Microbiol. Spectr. https://doi.org/10.1128/ microbiolspec. El 10-0009-2015 (2016).

125. El Zowalaty, M. E. et al. Pseudomonas aeruginosa: arsenal of resistance mechanisms, decades of changing resistance profiles, and future antimicrobial therapies. Future Microbiol. 10, 1683-1706 (2015).

126. Munoz-Price, L. S. et al. Clinical epidemiology of the global expansion of Klebsiella pneumoniae carbapenemases. Lancet Infect. Dis. 13, 785-796 (2013).

127. Dyson, Z. A., Klemm, E. J., Palmer, S. \& Dougan, G Antibiotic resistance and typhoid. Clin. Infect. Dis. 68 S165-s170 (2019)

128. Qamar, F. N., Azmatullah, A., Kazi, A. M., Khan, E. \& Zaidi, A. K. A three-year review of antimicrobial resistance of Salmonella enterica serovars Typhi and Paratyphi A in Pakistan. J. Infect. Dev. Ctries. 8 981-986 (2014)

129. Kariuki, S., Gordon, M. A., Feasey, N. \& Parry, C. M. Antimicrobial resistance and management of invasive Salmonella disease. Vaccine 33, C21-C29 (2015)

130. Gordon, M. A. et al. Epidemics of invasive Salmonella enterica serovar enteritidis and S. enterica Serovar typhimurium infection associated with multidrug resistance among adults and children in Malawi. Clin. Infect. Dis. 46, 963-969 (2008).

131. Puzari, M., Sharma, M. \& Chetia, P. Emergence of antibiotic resistant Shigella species: a matter of concern. J. Infect. Public Health 11, 451-454 (2018).

132. Zafar, A. et al. Antibiotic susceptibility in Streptococcus pneumoniae, Haemophilus influenzae and Streptococcus pyogenes in Pakistan: a review of results from the Survey of Antibiotic Resistance (SOAR) 2002-15. J. Antimicrob. Chemother. 71, i103-i109 (2016).

133. US Department of Health and Human Services. Antibiotic resistance threats in the United States. https://www.cdc.gov/drugresistance/pdf/ar-threats2013-508.pdf (2013)

134. Gandhi, N. R. et al. Multidrug-resistant and extensively drug-resistant tuberculosis: a threat to global control of tuberculosis. Lancet 375 , 1830-1843 (2010).

135. Nguyen, L. Antibiotic resistance mechanisms in M. tuberculosis: an update. Arch. Toxicol. 90 , 1585-1604 (2016).

136. Marston, H. D., Paules, C. I. \& Fauci, A. S. Monoclonal antibodies for emerging infectious diseases - borrowing from history. N. Engl. J. Med. 378, 1469-1472 (2018).

137. McConnell, M. J. Where are we with monoclonal antibodies for multidrug-resistant infections? Drug Discov. Today 24, 1132-1138 (2019).

138. DiGiandomenico, A. et al. A multifunctional bispecific antibody protects against Pseudomonas aeruginosa. Sci. Transl. Med. 6, 262 ra155 (2014).

139. Lowy, I. et al. Treatment with monoclonal antibodies against Clostridium difficile toxins. N. Engl. J. Med. 362, 197-205 (2010).

140. Gulati, S. et al. Complement alone drives efficacy of a chimeric antigonococcal monoclonal antibody. PLoS Biol. 17, e3000323 (2019).

141. Fischetti, V. A. Development of phage lysins as novel therapeutics: a historical perspective. Viruses 10, 310 (2018).

142. Merabishvili, M. et al. Quality-controlled small-scale production of a well-defined bacteriophage cocktail for use in human clinical trials. PLOS ONE 4, e4944 (2009).

143. Sahota, J. S. et al. Bacteriophage delivery by nebulization and efficacy against phenotypically diverse Pseudomonas aeruginosa from cystic fibrosis patients. J. Aerosol Med. Pulm. Drug Deliv. 28, 353-360 (2015).

144. Nale, J. Y. et al. Bacteriophage combinations significantly reduce Clostridium difficile growth in vitro and proliferation in vivo. Antimicrob. Agents Chemother. 60, 968-981 (2016).

145. Lima, R., Del Fiol, F. S. \& Balcao, V. M. Prospects for the use of new technologies to combat multidrug-resistant bacteria. Front. Pharmacol. 10, 692 (2019).

146. Relman, D. A. \& Lipsitch, M. Microbiome as a tool and a target in the effort to address antimicrobial resistance. Proc. Natl Acad. Sci. USA 115 12902-12910 (2018).

147. Khanna, S. et al. A novel microbiome therapeutic increases gut microbial diversity and prevents recurrent Clostridium difficile infection. J. Infect. Dis. 214, 173-181 (2016)

148. Gerding, D. N. et al. Administration of spores of nontoxigenic Clostridium difficile strain M3 for prevention of recurrent $\mathrm{C}$. difficile infection: a randomized clinical trial. JAMA 313, 1719-1727 (2015).

149. Kim, W. J. et al. Commensal Neisseria kill Neisseria gonorrhoeae through a DNA-dependent mechanism. Cell Host Microbe 26, 228-239.e8 (2019).

150. Idelevich, E. A. \& Becker, K. How to accelerate antimicrobial susceptibility testing. Clin. Microbiol. Infect. 25, 1347-1355 (2019).

151. van Belkum, A. et al. Developmental roadmap for antimicrobial susceptibility testing systems. Nat. Rev. Microbiol. 17, 51-62 (2019).

152. Li, W. et al. Rapid identification and antimicrobial susceptibility testing for urinary tract pathogens by direct analysis of urine samples using a MALDI-TOF MS-based combined protocol. Front. Microbiol. 10 1182 (2019).

153. Rappuoli, R. Glycoconjugate vaccines: principles and mechanisms. Sci. Transl. Med. 10, eaat4615 (2018)

154. Delany, I., Rappuoli, R. \& De Gregorio, E. Vaccines for the 21 st century. EMBO Mol. Med. 6, 708-720 (2014).

155. Serruto, D., Serino, L., Masignani, V \& Pizza, M. Genome-based approaches to develop vaccines against bacterial pathogens. Vaccine 27, 3245-3250 (2009). 


\section{REVIEWS}

156. Serruto, D., Bottomley, M. J., Ram, S., Giuliani, M. M. $\&$ Rappuoli, R. The new multicomponent vaccine against meningococcal serogroup B, 4CMenB: immunological, functional and structural characterization of the antigens. Vaccine 30, B87-B97 (2012).

157. Moriel, D. G et al. Identification of protective and broadly conserved vaccine antigens from the genome of extraintestinal pathogenic Escherichia coli. Proc. Natl Acad. Sci. USA 107, 9072-9077 (2010).

158. Rappuoli, R., Bottomley, M. J., D'Oro, U., Finco, O. $\&$ De Gregorio, E. Reverse vaccinology 2.0: human immunology instructs vaccine antigen design. J. Exp. Med. 213, 469-481 (2016).

159. Crank, M. C. et al. A proof of concept for structurebased vaccine design targeting RSV in humans. Science 365, 505-509 (2019).

160. Scarselli, M. et al. Rational design of a meningococcal antigen inducing broad protective immunity. Sci. Transl. Med. 3, 91 ra62 (2011).

161. Gnopo, Y. M. D., Watkins, H. C., Stevenson, T. C. DeLisa, M. P. \& Putnam, D. Designer outer membrane vesicles as immunomodulatory systems-reprogramming bacteria for vaccine delivery. Adv. Drug Deliv. Rev. 114, 132-142 (2017)

162. Gerke, C. et al. Production of a shigella sonnei vaccine based on generalized modules for membrane antigens (GMMA), 1790GAHB. PLOS ONE 10, e0134478 (2015).

163. Launay, O. et al. Safety profile and immunologic responses of a novel vaccine against Shigella sonnei administered intramuscularly, intradermally and intranasally: results from two parallel randomized phase 1 clinical studies in healthy adult volunteers in Europe. EBioMedicine 22, 164-172 (2017).

164. Launay, O. et al. Booster vaccination with GVGH Shigella sonnei 1790GAHB GMMA vaccine compared to single vaccination in unvaccinated healthy European adults: results from a phase 1 clinical trial. Front. Immunol. 10, 335 (2019).

165. Wacker, M. et al. N-linked glycosylation in Campylobacter jejuni and its functional transfer into E. coli. Science 298, 1790-1793 (2002).

166. Kampf, M. M. et al. In vivo production of a novel glycoconjugate vaccine against Shigella flexneri $2 a$ in recombinant Escherichia coli: identification of stimulating factors for in vivo glycosylation. Microb. Cell Fact. 14, 12 (2015).

167. Reed, S. G., Orr, M. T. \& Fox, C. B. Key roles of adjuvants in modern vaccines. Nat. Med. 19 1597-1608 (2013).

168. Otieno, L. et al. Safety and immunogenicity of RTS,S/AS01 malaria vaccine in infants and children with WHO stage 1 or 2 HIV disease: a randomised, double-blind, controlled trial. Lancet Infect. Dis. 16 1134-1144 (2016)

169. Cunningham, A. L. et al. Efficacy of the herpes zoster subunit vaccine in adults 70 years of age or older. N. Engl. J. Med. 375, 1019-1032 (2016).

\section{Author contributions}

F.M., F.B. and D.S. researched data for the article and wrote the article. All authors substantially contributed to the discussion of the content, and reviewed and edited the manuscript before submission.

\section{Competing interests}

F.M., F.B., R.R. and D.S. are employees of the GSK group of companies, which is involved in the discovery and commercialization of vaccines and therapeutics against bacterial infections.

Peer review information

Nature Reviews Microbiology thanks K. Atkins and S. Vong for their contribution to the peer review of this work.

Publisher's note

Springer Nature remains neutral with regard to jurisdictional claims in published maps and institutional affiliations.

\section{Supplementary information}

Supplementary information is available for this paper at https://doi.org/10.1038/s41579-020-00506-3.

\section{RELATED LINKS}

AMR Action Fund: https://amractionfund.com.

CDC: https://www.cdc.gov/hai/patientsafety/ar-hospitals.html

FAO Global Forum on Food Security and Nutrition:

http://www.fao.org/3/cb0863en/cb0863en.pdf

Institute for Health Metrics and Evaluation:

http://www.healthdata.org/gbd

(C) Springer Nature Limited 2021 